\title{
Deep Scattering Spectrum
}

\author{
Joakim Andén, Student Member, IEEE, Stéphane Mallat, Fellow, IEEE
}

\begin{abstract}
A scattering transform defines a locally translation invariant representation which is stable to time-warping deformations. It extends MFCC representations by computing modulation spectrum coefficients of multiple orders, through cascades of wavelet convolutions and modulus operators. Second-order scattering coefficients characterize transient phenomena such as attacks and amplitude modulation. A frequency transposition invariant representation is obtained by applying a scattering transform along log-frequency. State-the-of-art classification results are obtained for musical genre and phone classification on GTZAN and TIMIT databases, respectively.
\end{abstract}

Index Terms-Audio classification, deep neural networks, MFCC, modulation spectrum, wavelets.

\section{INTRODUCTION}

A major difficulty of audio representations for classification is the multiplicity of information at different time scales: pitch and timbre at the scale of milliseconds, the rhythm of speech and music at the scale of seconds, and the music progression over minutes and hours. Mel-frequency cepstral coefficients (MFCCs) are efficient local descriptors at time scales up to $25 \mathrm{~ms}$. Capturing larger structures up to $500 \mathrm{~ms}$ is however necessary in most applications. This paper studies the construction of stable, invariant signal representations over such larger time scales. We concentrate on audio applications, but introduce a generic scattering representation for classification, which applies to many signal modalities beyond audio [1].

Spectrograms compute locally time-shift invariant descriptors over durations limited by a window. However, Section [II shows that high-frequency spectrogram coefficients are not stable to variability due to time-warping deformations, which occur in most signals, particularly in audio. Stability means that small signal deformations produce small modifications of the representation, measured with a Euclidean norm. This is particularly important for classification. Mel-frequency spectrograms are obtained by averaging spectrogram values over mel-frequency bands. It improves stability to time warping, but it also removes information. Over time intervals larger than $25 \mathrm{~ms}$, the information loss becomes too important, which is why mel-frequency spectrograms and MFCCs, are limited to such short time intervals. Modulation spectrum decompositions [2]-[10] characterize the temporal evolution of mel-frequency spectrograms over larger time scales, with autocorrelation or Fourier coefficients. However, this modulation spectrum also suffers from instability to time-warping deformation, which impedes classification performance.

Section III shows that the information lost by mel-frequency spectrograms can be recovered with multiple layers of wavelet

This work is supported by the ANR 10-BLAN-0126 and ERC InvariantClass 320959 grants. coefficients. In addition to being locally invariant to timeshifts, this representation is also stable to time-warping deformation. Known as a scattering transform [11], it is computed through a cascade of wavelet transforms and modulus nonlinearities. The computational structure is similar to a convolutional deep neural network [12]-[19], but involves no learning. It outputs time-averaged coefficients, providing informative signal invariants over potentially large time scales.

A scattering transform has striking similarities with physiological models of the cochlea and of the auditory pathway [20], [21], also used for audio processing [22]. Its energy conservation and other mathematical properties are reviewed in Section IV. An approximate inverse scattering transform is introduced in Section V, with numerical examples. Section VI relates the amplitude of scattering coefficients to audio signal properties. These coefficients provide accurate measurements of frequency intervals between harmonics and also characterize the amplitude modulation of voiced and unvoiced sounds. The logarithm of scattering coefficients linearly separates audio components related to pitch, formant and timbre.

Frequency transpositions form another important source of audio variability, which should be kept or removed depending upon the classification task. For example, speaker-independent phone classification requires some frequency transposition invariance, while frequency localization is necessary for speaker identification. Section VII shows that cascading a scattering transform along log-frequency yields a transposition invariant representation which is stable to frequency deformation.

Scattering representations have proved useful for image classification [23], [24], where spatial translation invariance is crucial. In audio, the analogous time-shift invariance is also important, but scattering transforms are computed with very different wavelets. They have a better frequency resolution, which is adapted to audio frequency structures. Section VIII explains how to adapt and optimize the frequency invariance for each signal class at the supervised learning stage. A time and frequency scattering representation is used for musical genre classification over the GTZAN database, and for phone segment classification over the TIMIT corpus. State-of-theart results are obtained with a Gaussian kernel SVM applied to scattering feature vectors. All figures and results are reproducible using a MATLAB software package, available at http://www.di.ens.fr/data/scattering/.

\section{MEL-FrEQUENCY SPECTRUM}

Section III-A shows that high-frequency spectrogram coefficients are not stable to time-warping deformation. The mel-frequency spectrogram stabilizes these coefficients by averaging them along frequency, but loses information. To analyze this information loss, Section $\mathrm{II}-\mathrm{B}$ relates the mel- 

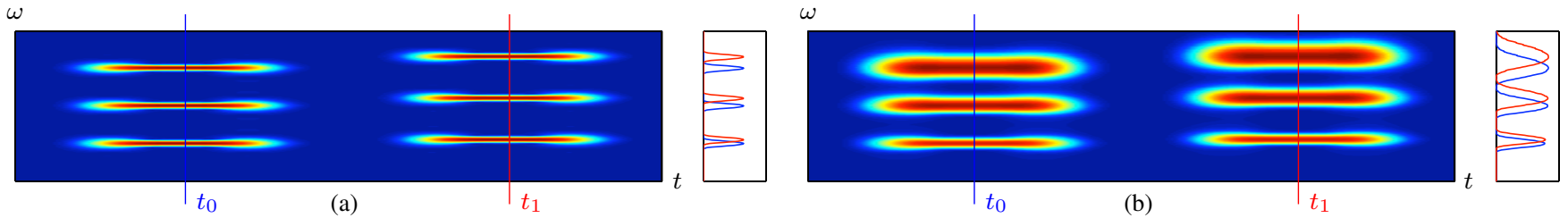

Fig. 1. (a) Spectrogram $\log |\widehat{x}(t, \omega)|$ for a harmonic signal $x(t)$ (centered in $t_{0}$ ) followed by $\log \left|\widehat{x}_{\tau}(t, \omega)\right|$ for $x_{\tau}(t)=x((1-\epsilon) t)$ (centered in $t_{1}$ ), as a function of $t$ and $\omega$. The right graph plots $\log \left|\widehat{x}\left(t_{0}, \omega\right)\right|$ (blue) and $\log \left|\widehat{x}_{\tau}\left(t_{1}, \omega\right)\right|$ (red) as a function of $\omega$. Their partials do not overlap at high frequencies. (b) Mel-frequency spectrogram $\log M x(t, \omega)$ followed by $\log M x_{\tau}(t, \omega)$. The right graph plots $\log M x\left(t_{0}, \omega\right)$ (blue) and $\log M x_{\tau}\left(t_{1}, \omega\right)$ (red) as a function of $\omega$. With a mel-scale frequency averaging, the partials of $x$ and $x_{\tau}$ overlap at all frequencies.

frequency spectrogram to the amplitude output of a filter bank which computes a wavelet transform.

\section{A. Fourier Invariance and Deformation Instability}

Let $\widehat{x}(\omega)=\int x(u) e^{-i \omega u} d u$ be the Fourier transform of $x$. If $x_{c}(t)=x(t-c)$ then $\widehat{x}_{c}(\omega)=e^{-i c \omega} \widehat{x}(\omega)$. The Fourier transform modulus is thus invariant to translation:

$$
\left|\widehat{x}_{c}(\omega)\right|=|\widehat{x}(\omega)| \text {. }
$$

A spectrogram localizes this translation invariance with a window $\phi$ of duration $T$ such that $\int \phi(u) d u=1$. It is defined by

$$
|\widehat{x}(t, \omega)|=\left|\int x(u) \phi(u-t) e^{-i \omega u} d u\right| .
$$

If $|c| \ll T$ then one can verify that $\left|\widehat{x}_{c}(t, \omega)\right| \approx|\widehat{x}(t, \omega)|$.

However, invariance to time-shifts is often not enough. Suppose that $x$ is not just translated but time-warped to give $x_{\tau}(t)=x(t-\tau(t))$ with $\left|\tau^{\prime}(t)\right|<1$. A representation $\Phi(x)$ is said to be stable to deformation if its Euclidean norm $\left\|\Phi(x)-\Phi\left(x_{\tau}\right)\right\|$ is small when the deformation is small. The deformation size is measured by $\sup _{t}\left|\tau^{\prime}(t)\right|$. If it vanishes then it is a "pure" translation without deformation. Stability is formally defined as a Lipschitz continuity condition relatively to this metric. It means that there exists $C>0$ such that for $x(t)$ and all $\tau$ with $\sup _{t}\left|\tau^{\prime}(t)\right|<1$

$$
\left\|\Phi(x)-\Phi\left(x_{\tau}\right)\right\| \leq C \sup _{t}\left|\tau^{\prime}(t)\right|\|x\| .
$$

The constant $C$ is a measure of stability.

This Lipschitz continuity property implies that time-warping deformations are locally linearized by $\Phi(x)$. Indeed, Lipschitz continuous operators are almost everywhere differentiable. It results that $\Phi(x)-\Phi\left(x_{\tau}\right)$ can be approximated by a linear operator if $\sup _{t}\left|\tau^{\prime}(t)\right|$ is small. A family of small deformations thus generate a linear space. In the transformed space, an invariant to these deformations can then be computed with a linear projector on the orthogonal complement to this linear space. In Section VIII we use linear discriminant classifiers to become selectively invariant to small time-warping deformations.

A Fourier modulus representation $\Phi(x)=|\widehat{x}|$ is not stable to deformation because high frequencies are severely distorted by small deformations. For example, let us consider a small dilation $\tau(t)=\epsilon t$ with $0<\epsilon \ll 1$. Since $\tau^{\prime}(t)=\epsilon$, the Lipschitz continuity condition (3) becomes

$$
\left\||\widehat{x}|-\left|\widehat{x_{\tau}}\right|\right\| \leq C \epsilon\|x\| .
$$

The Fourier transform of $x_{\tau}(t)=x((1-\epsilon) t)$ is $\widehat{x}_{\tau}(\omega)=$ $(1-\epsilon)^{-1} \widehat{x}\left((1-\epsilon)^{-1} \omega\right)$. This dilation shifts a frequency component at $\omega_{0}$ by $\epsilon\left|\omega_{0}\right|$. For a harmonic signal $x(t)=$ $g(t) \sum_{n} a_{n} \cos (n \xi t)$, the Fourier transform is a sum of partials

$$
\widehat{x}(\omega)=\sum_{n} \frac{a_{n}}{2}(\widehat{g}(\omega-n \xi)+\widehat{g}(\omega+n \xi)) .
$$

After time-warping, each partial $\widehat{g}(\omega \pm n \xi)$ is translated by $\epsilon n \xi$, as shown in the spectrogram of Figure 11a). Even though $\epsilon$ is small, at high frequencies $n \epsilon \xi$ becomes larger than the bandwidth of $\widehat{g}$. Consequently, the harmonics $\widehat{g}\left(\omega(1-\epsilon)^{-1}-n \xi\right)$ of $\widehat{x}_{\tau}$ do not overlap with the harmonics $\widehat{g}(\omega-n \xi)$ of $\widehat{x}$. The Euclidean distance of $|\widehat{x}|$ and $\left|\widehat{x}_{\tau}\right|$ thus does not decrease proportionally to $\epsilon$ if the harmonic amplitudes $a_{n}$ are sufficiently large at high frequencies. This proves that the deformation stability condition (4) is not satisfied for any $C>0$.

The autocorrelation $R x(u)=\int x(t) x^{\star}(t-u) d t$ is also a translation invariant representation which has the same deformation instability as the Fourier transform modulus. Indeed, $\widehat{R} x(\omega)=|\widehat{x}(\omega)|^{2}$ so $\left\|R x-R x_{\tau}\right\|=(2 \pi)^{-1}\left\||\widehat{x}|^{2}-\left|\widehat{x}_{\tau}\right|^{2}\right\|$.

\section{B. Mel-frequency Deformation Stability and Filter Banks}

A mel-frequency spectrogram averages the spectrogram energy with mel-scale filters $\widehat{\psi}_{\lambda}$, where $\lambda$ is the center frequency of each $\widehat{\psi}_{\lambda}(\omega)$ :

$$
\mathrm{M} x(t, \lambda)=\frac{1}{2 \pi} \int|\widehat{x}(t, \omega)|^{2}\left|\widehat{\psi}_{\lambda}(\omega)\right|^{2} d \omega .
$$

The band-pass filters $\widehat{\psi}_{\lambda}$ have a constant- $Q$ frequency bandwidth at high frequencies. Their frequency support is centered at $\lambda$ with a bandwidth of the order of $\lambda / Q$. At lower frequencies, instead of being constant-Q, the bandwidth of $\widehat{\psi}_{\lambda}$ remains equal to $2 \pi / T$.

The mel-frequency averaging removes deformation instability created by large displacements of high frequencies under dilations. If $x_{\tau}(t)=x((1-\epsilon) t)$ then we saw that each frequency component at $\omega_{0}$ is moved by $\epsilon\left|\omega_{0}\right|$, which may be large if $\left|\omega_{0}\right|$ is large. However, the mel-scale filter $\widehat{\psi}_{\lambda}(\omega)$ covering the frequency $\omega_{0}$ has a frequency bandwidth of the order of $\lambda / Q \sim\left|\omega_{0}\right| / Q$. As a result, the relative error after averaging by $|\widehat{\psi}|^{2}$ is of the order of $\epsilon Q$. This is illustrated by Figure 1.b) on a harmonic signal $x$. After mel-frequency averaging, the frequency partials of $x$ and $x_{\tau}$ overlap at all frequencies. One can verify that $\left\|M x(t, \lambda)-M_{\tau} x(t, \lambda)\right\| \leq C \epsilon\|x\|$, where $C$ is proportional to $Q$, and does not depend upon $\epsilon$ and $x$. 


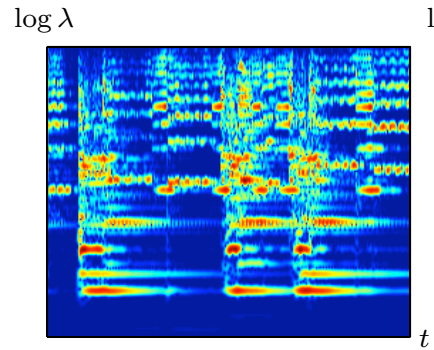

(a)

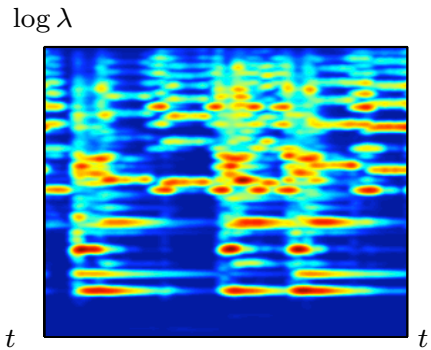

(b)
Fig. 2. (a): Scalogram $\log \left|x \star \psi_{\lambda}(t)\right|^{2}$ for a musical signal, as a function of $t$ and $\lambda$. (b): Averaged scalogram $\log \left|x \star \psi_{\lambda}\right|^{2} \star \phi^{2}(t)$ with a lowpass filter $\phi$ of duration $T=190 \mathrm{~ms}$.

Unlike the spectrogram (2), the mel-frequency spectrogram (6) satisfies the Lipschitz deformation stability condition (3).

Mel-scale averaging provides time-warping stability but loses information. We show that this frequency averaging is equivalent to a time averaging of a filter bank output, which will provide a strategy to recover the lost information. Since $\widehat{x}(t, \omega)$ in (2) is the Fourier transform of $x_{t}(u)=x(u) \phi(u-t)$, applying Plancherel's formula gives

$$
\begin{aligned}
\mathrm{M} x(t, \lambda) & =\frac{1}{2 \pi} \int\left|\widehat{x}_{t}(\omega)\right|^{2}\left|\widehat{\psi}_{\lambda}(\omega)\right|^{2} d \omega \\
& =\int\left|x_{t} \star \psi_{\lambda}(v)\right|^{2} d v \\
& =\int\left|\int x(u) \phi(u-t) \psi_{\lambda}(v-u) d u\right|^{2} d v
\end{aligned}
$$

If $\lambda \gg Q / T$ then $\phi(t)$ is approximately constant on the support of $\psi_{\lambda}(t)$, so $\phi(u-t) \psi_{\lambda}(v-u) \approx \phi(v-t) \psi_{\lambda}(v-u)$, and hence

$$
\begin{aligned}
\mathrm{M} x(t, \lambda) & \approx \int\left|\int x(u) \psi_{\lambda}(v-u) d u\right|^{2}|\phi(v-t)|^{2} d v \\
& =\left|x \star \psi_{\lambda}\right|^{2} \star|\phi|^{2}(t) .
\end{aligned}
$$

The frequency averaging of the spectrogram is thus nearly equal to the time averaging of $\left|x \star \psi_{\lambda}\right|^{2}$. In this formulation, the window $\phi$ acts as a lowpass filter, ensuring that the representation is locally invariant to time-shifts smaller than $T$. Section III-A studies the properties of the constant-Q filter bank $\left\{\psi_{\lambda}\right\}_{\lambda}$, which defines an analytic wavelet transform.

Figures 2(a) and 2(b) display $\left|x \star \psi_{\lambda}\right|^{2}$ and $\left|x \star \psi_{\lambda}\right|^{2} \star|\phi|^{2}$, respectively, for a musical recording. The window duration is $T=190 \mathrm{~ms}$. This time averaging removes fine-scale information such as vibratos and attacks. To reduce information loss, a mel-frequency spectrogram is often computed over small time windows of about $25 \mathrm{~ms}$. As a result, it does not capture large-scale structures, which limits classification performance.

To increase $T$ without losing too much information, it is necessary to capture the amplitude modulations of $\left|x \star \psi_{\lambda}(t)\right|$ at scales smaller than $T$, which are important in audio perception. The spectrum of these modulation envelopes can be computed from the spectrogram [2]-[5] of $\left|x \star \psi_{\lambda}\right|$, or represented with a short-time autocorrelation [6], [7]. However, these modulation spectra are unstable to time-warping deformations. Indeed, a time-warping of $x$ induces a time-warping of $\left|x \star \psi_{\lambda}\right|$, and
Section II-A showed that spectrograms and autocorrelations have deformation instabilities. Constant-Q averaged modulation spectra [9], [10] stabilize spectrogram representations with another averaging along modulation frequencies. According to (11), this can also be computed with a second constant-Q filter bank. The scattering transform follows this latter approach.

\section{WAVELET SCATTERING TRANSFORM}

A scattering transform recovers the information lost by a mel-frequency averaging with a cascade of wavelet decompositions and modulus operators [11]. It is locally translation invariant and stable to time-warping deformation. Important properties of constant-Q filter banks are first reviewed in the framework of a wavelet transform, and the scattering transform is introduced in Section III-B

\section{A. Analytic Wavelet Transform and Modulus}

Constant-Q filter banks compute a wavelet transform. We review the properties of complex analytic wavelet transforms and their modulus, which are used to calculate mel-frequency spectral coefficients.

A wavelet $\psi(t)$ is a band-pass filter with $\widehat{\psi}(0)=0$. We consider complex wavelets with quadrature phase such that $\widehat{\psi}(\omega) \approx 0$ for $\omega<0$. For any $\lambda>0$, a dilated wavelet of center frequency $\lambda$ is written

$$
\psi_{\lambda}(t)=\lambda \psi(\lambda t) \text { and hence } \widehat{\psi}_{\lambda}(\omega)=\widehat{\psi}\left(\frac{\omega}{\lambda}\right) .
$$

The center frequency of $\widehat{\psi}$ is normalized to 1 . In the following, we denote by $Q$ the number of wavelets per octave, which means that $\lambda=2^{k / Q}$ for $k \in \mathbb{Z}$. The bandwidth of $\widehat{\psi}$ is of the order of $Q^{-1}$, to cover the whole frequency axis with these band-pass wavelet filters. The support of $\widehat{\psi}_{\lambda}(\omega)$ is centered in $\lambda$ with a frequency bandwidth $\lambda / Q$ whereas the energy of $\psi_{\lambda}(t)$ is concentrated around 0 in an interval of size $2 \pi Q / \lambda$. To guarantee that this interval is smaller than $T$, we define $\psi_{\lambda}$ with 12 only for $\lambda \geq 2 \pi Q / T$. For $\lambda<2 \pi Q / T$, the lowerfrequency interval $[0,2 \pi Q / T]$ is covered with about $Q-1$ equally-spaced filters $\widehat{\psi}_{\lambda}$ with constant frequency bandwidth $2 \pi / T$. For simplicity, these lower-frequency filters are still called wavelets. We denote by $\Lambda$ the grid of all wavelet center frequencies $\lambda$.

The wavelet transform of $x$ computes a convolution of $x$ with a low-pass filter $\phi$ of frequency bandwidth $2 \pi / T$, and convolutions with all higher-frequency wavelets $\psi_{\lambda}$ for $\lambda \in \Lambda$ :

$$
W x=\left(x \star \phi(t), x \star \psi_{\lambda}(t)\right)_{t \in \mathbb{R}, \lambda \in \Lambda} .
$$

This time index $t$ is not critically sampled as in wavelet bases so this representation is highly redundant. The wavelet $\psi$ and the low-pass filter $\phi$ are designed to build filters which cover the whole frequency axis, which means that

$$
A(\omega)=|\widehat{\phi}(\omega)|^{2}+\frac{1}{2} \sum_{\lambda \in \Lambda}\left(\left|\widehat{\psi}_{\lambda}(\omega)\right|^{2}+\left|\widehat{\psi}_{\lambda}(-\omega)\right|^{2}\right)
$$

satisfies, for all $\omega \in \mathbb{R}$ :

$$
1-\alpha \leq A(\omega) \leq 1 \text { with } \alpha<1 .
$$


This condition implies that the wavelet transform $W$ is a stable and invertible operator. Multiplying $(15)$ by $|\widehat{x}(\omega)|^{2}$ and applying the Plancherel formula [25] gives

$$
(1-\alpha)\|x\|^{2} \leq\|W x\|^{2} \leq\|x\|^{2},
$$

where $\|x\|^{2}=\int|x(t)|^{2} d t$ and where the squared norm of $W x$ sums all squared coefficients:

$$
\|W x\|^{2}=\int|x \star \phi(t)|^{2} d t+\sum_{\lambda \in \Lambda} \int\left|x \star \psi_{\lambda}(t)\right|^{2} d t .
$$

The upper bound (16) means that $W$ is a contractive operator and the lower bound implies that it has a stable inverse. One can also verify that the pseudo-inverse of $W$ recovers $x$ with the following formula

$$
x(t)=(x \star \phi) \star \bar{\phi}(t)+\sum_{\lambda \in \Lambda} \operatorname{Real}\left(\left(x \star \psi_{\lambda}\right) \star \bar{\psi}_{\lambda}(t)\right),
$$

with reconstruction filters defined by

$$
\widehat{\bar{\phi}}(\omega)=\frac{\widehat{\phi}^{*}(\omega)}{A(\omega)} \text { and } \widehat{\bar{\psi}}_{\lambda}(\omega)=\frac{\widehat{\psi}_{\lambda}^{*}(\omega)}{A(\omega)},
$$

where $z^{*}$ is the complex conjugate of $z \in \mathbb{C}$. If $\alpha=0$ in (15) then $W$ is said to be a tight frame operator, in which case $\bar{\phi}(t)=\phi(-t)$ and $\bar{\psi}_{\lambda}(t)=\psi_{\lambda}^{*}(-t)$.

One may define an analytic wavelet with an octave resolution $Q$ as $\psi(t)=e^{i t} \theta(t)$ and hence $\widehat{\psi}(\omega)=\widehat{\theta}(\omega-1)$ where $\hat{\theta}$ is the transfer function of a low-pass filter whose bandwidth is of the order of $Q^{-1}$. If $\widehat{\theta}(-1) \neq 0$ then we define $\widehat{\psi}(\omega)=\widehat{\theta}(\omega-1)-\widehat{\theta}(\omega) \hat{\theta}(-1) / \widehat{\theta}(0)$, which guarantees that $\widehat{\psi}(0)=0$. If $\theta$ is a Gaussian then $\psi$ is called a Morlet wavelet, which is almost analytic because $|\widehat{\psi}(\omega)|$ is small but not strictly zero for $\omega<0$. Figure 3 shows Morlet wavelets $\widehat{\psi}_{\lambda}$ with $Q=8$. In this case $\phi$ is also chosen to be a Gaussian. For $Q=1$, tight frame wavelet transforms can also be obtained by choosing $\psi$ to be the analytic part of a real wavelet which generates an orthogonal wavelet basis, such as a cubic spline wavelet [11]. Unless indicated otherwise, wavelets used in this paper are Morlet wavelets.

Following (11), mel-frequency spectrograms can be approximated using a non-linear wavelet modulus operator which removes the complex phase of all wavelet coefficients:

$$
|W| x=\left(x \star \phi(t),\left|x \star \psi_{\lambda}(t)\right|\right)_{t \in \mathbb{R}, \lambda \in \Lambda} .
$$

Taking the modulus of analytic wavelet coefficient can be interpreted as a sub-band Hilbert envelope demodulation. Demodulation is used to separate carriers and modulation envelopes. When a carrier or pitch frequency can be detected, then a linear coherent demodulation is efficiently implemented by multiplying the analytic signal with the conjugate of the detected carrier [26]-[28]. However, many signals such as unvoiced speech are not modulated by isolated carrier frequency, in which case coherent demodulation is not well defined. Nonlinear Hilbert envelope demodulations apply to any band-pass analytic signals, but if a carrier is present then the Hilbert envelope depends both on the carrier and on the amplitude modulation. Section VI-C explains how to isolate amplitude

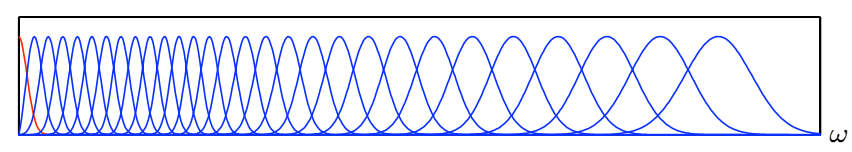

Fig. 3. Morlet wavelets $\widehat{\psi}_{\lambda}(\omega)$ with $Q=8$ wavelets per octave, for different $\lambda$. The low-frequency filter $\widehat{\phi}(\omega)$ (in red) is a Gaussian.

modulation coefficients from Hilbert envelope measurements, whether a carrier is present or not.

Although a wavelet modulus operator removes the complex phase, it does not lose information because the temporal variation of the multiscale envelopes is kept. A signal cannot be reconstructed from the modulus of its Fourier transform, but it can be recovered from the modulus of its wavelet transform. Since the time variable $t$ is not subsampled, a wavelet transform has more coefficients than the original signal. These coefficients are highly redundant when filters have a significant frequency overlap. For particular families of analytic wavelets, one can prove that $|W|$ is an invertible operator with a continuous inverse [29]. This is further studied in Section $\mathrm{V}$.

The operator $|W|$ is contractive. Indeed, the wavelet transform $W$ is contractive and the complex modulus is contractive in the sense that ||$a|-| b|| \leq|a-b|$ for any $(a, b) \in \mathbb{C}^{2}$ so

$$
\left\||W| x-|W| x^{\prime}\right\|^{2} \leq\left\|W x-W x^{\prime}\right\|^{2} \leq\left\|x-x^{\prime}\right\|^{2} .
$$

If $W$ is a tight frame operator then $\||W| x\|=\|W x\|=\|x\|$ so $|W|$ preserves the signal norm.

\section{B. Deep Scattering Network}

We showed in (11) that mel-frequency spectral coefficients $M x(t, \lambda)$ are approximately equal to averaged squared wavelet coefficients $\left|x \star \psi_{\lambda}\right|^{2} \star|\phi|^{2}(t)$. Large wavelet coefficients are considerably amplified by the square operator. To avoid amplifying outliers, we remove the square and calculate $\left|x \star \psi_{\lambda}\right| \star \phi(t)$ instead. High frequencies removed by the lowpass filter $\phi$ are recovered by a new set of wavelet modulus coefficients. Cascading this procedure defines a scattering transform.

A locally translation invariant descriptors of $x$ is obtained with a time-average $S_{0} x(t)=x \star \phi(t)$, which removes all high frequencies. These high-frequencies are recovered by a wavelet modulus transform

$$
\left|W_{1}\right| x=\left(x \star \phi(t),\left|x \star \psi_{\lambda_{1}}(t)\right|\right)_{t \in \mathbb{R}, \lambda_{1} \in \Lambda_{1}} .
$$

It is computed with wavelets $\psi_{\lambda_{1}}$ having an octave frequency resolution $Q_{1}$. For audio signals we set $Q_{1}=8$, which defines wavelets having the same frequency resolution as melfrequency filters. Audio signals have little energy at low frequencies so $S_{0} x(t) \approx 0$. Approximate mel-frequency spectral coefficients are obtained by averaging the wavelet modulus coefficients with $\phi$ :

$$
S_{1} x\left(t, \lambda_{1}\right)=\left|x \star \psi_{\lambda_{1}}\right| \star \phi(t) .
$$

These are called first-order scattering coefficients. They are computed with a second wavelet modulus transform $\left|W_{2}\right|$ 
applied to each $\left|x \star \psi_{\lambda_{1}}\right|$, which also provides complementary high-frequency wavelet coefficients:

$$
\left|W_{2}\right|\left|x \star \psi_{\lambda_{1}}\right|=\left(\left|x \star \psi_{\lambda_{1}}\right| \star \phi,|| x \star \psi_{\lambda_{1}}\left|\star \psi_{\lambda_{2}}\right|\right)_{\lambda_{2} \in \Lambda_{2}} .
$$

The wavelets $\psi_{\lambda_{2}}$ have an octave resolution $Q_{2}$ which may be different from $Q_{1}$. It is chosen to get a sparse representation which means concentrating the signal information over as few wavelet coefficients as possible. These coefficients are averaged by the lowpass filter $\phi$ of size $T$, which ensures local invariance to time-shifts, as with the first-order coefficients. It defines second-order scattering coefficients:

$$
S_{2} x\left(t, \lambda_{1}, \lambda_{2}\right)=|| x \star \psi_{\lambda_{1}}\left|\star \psi_{\lambda_{2}}\right| \star \phi(t) .
$$

These averages are computed by applying a third wavelet modulus transform $\left|W_{3}\right|$ to each ||$x \star \psi_{\lambda_{1}}\left|\star \psi_{\lambda_{2}}\right|$. It computes their wavelet modulus coefficients through convolutions with a new set of wavelets $\psi_{\lambda_{3}}$ having an octave resolution $Q_{3}$. Iterating this process defines scattering coefficients at any order $m$.

For any $m \geq 1$, iterated wavelet modulus convolutions are written:

$$
U_{m} x\left(t, \lambda_{1}, \ldots, \lambda_{m}\right)=||\left|x \star \psi_{\lambda_{1}}\right| \star \ldots\left|\star \psi_{\lambda_{m}}(t)\right|,
$$

where $m$ th-order wavelets $\psi_{\lambda_{m}}$ have an octave resolution $Q_{m}$, and satisfy the stability condition (15). Averaging $U_{m} x$ with $\phi$ gives scattering coefficients of order $m$ :

$$
\begin{aligned}
S_{m} x\left(t, \lambda_{1}, \ldots, \lambda_{m}\right) & =||\left|x \star \psi_{\lambda_{1}}\right| \star \ldots\left|\star \psi_{\lambda_{m}}\right| \star \phi(t) \\
& =U_{m} x\left(., \lambda_{1}, \ldots, \lambda_{m}\right) \star \phi(t) .
\end{aligned}
$$

Applying $\left|W_{m+1}\right|$ on $U_{m} x$ computes both $S_{m} x$ and $U_{m+1} x$ :

$$
\left|W_{m+1}\right| U_{m} x=\left(S_{m} x, U_{m+1} x\right) .
$$

A scattering decomposition of maximal order $l$ is thus defined by initializing $U_{0} x=x$, and recursively computing (22) for $0 \leq m \leq l$. This scattering transform is illustrated in Figure 4 The final scattering vector aggregates all scattering coefficients for $0 \leq m \leq l$ :

$$
S x=\left(S_{m} x\right)_{0 \leq m \leq l} .
$$

The scattering cascade of convolutions and non-linearities can also be interpreted as a convolutional network [12], where $U_{m} x$ is the set of coefficients of the $m$ th internal network layer. These networks have been shown to be highly effective for audio classification [13]-[19]. However, unlike standard convolutional networks, each such layer has an output $S_{m} x=U_{m} x \star \phi$, not just the last layer. In addition, all filters are predefined wavelets and are not learned from training data. A scattering transform, like MFCCs, provide a low-level invariant representation of the signal, without learning. It relies on prior information concerning the type of invariants that need to be computed, in this case relatively to time-shifts and time-warping deformations, or in Section VII relatively to frequency transpositions. When no such information is available, or if the sources of variability are much more complex, then it is necessary to learn them from examples, which is a task well suited for deep neural networks. In that sense both approaches are complementary.
The wavelet octave resolutions are optimized at each layer $m$ to produce sparse wavelet coefficients at the next layer. This better preserves the signal information as explained in Section $\mathrm{V}$. Sparsity seems also to play an important role for classification [30], [31]. For audio signals $x$, choosing $Q_{1}=8$ wavelets per octave has been shown to provide sparse representations of a mix of speech, music and environmental signals [32]. It nearly corresponds to a mel-scale frequency subdivision.

At the second order, choosing $Q_{2}=1$ defines wavelets with more narrow time support, which are better adapted to characterize transients and attacks. Section VI shows that musical signals including modulation structures such as tremolo may however require wavelets having better frequency resolution, and hence $Q_{2}>1$. At higher orders $m \geq 3$ we always set $Q_{m}=1$, but we shall see that these coefficients can often be neglected.

The scattering cascade has similarities with several neurophysiological models of auditory processing, which incorporate cascades of constant-Q filter banks followed by nonlinearities [20], [21]. The first filter bank with $Q_{1}=8$ models the cochlear filtering, whereas the second filter bank corresponds to later processing in the models with filters that have $Q_{2}=1$ [20], [21].

\section{Scattering Properties}

We briefly review important properties of scattering transforms, including stability to time-warping deformation, energy conservation, and describe a fast computational algorithm.

\section{A. Time-Warping Stability}

Stability to time-warping allows one to use linear operators for calculating invariant descriptors to small time-warping deformations. The Fourier transform is unstable to deformation because dilating a sinusoidal wave yields a new sinusoidal wave of different frequency which is orthogonal to the original one. Section II explains that mel-frequency spectrograms become stable to time-warping deformation with a frequency averaging. One can prove that a scattering representation $\Phi(x)=S x$ satisfies the Lipschitz continuity condition 3. because wavelets are stable to time-warping [11]. Let us write $\psi_{\lambda, \tau}(t)=\psi_{\lambda}(t-\tau(t))$. One can verify that there exists $C>0$ such that $\left\|\psi_{\lambda}-\psi_{\lambda, \tau}\right\| \leq C\left\|\psi_{\lambda}\right\| \sup _{t}\left|\tau^{\prime}(t)\right|$, for all $\lambda$ and all $\tau(t)$. This property is at the core of the scattering stability to time-warping deformations.

The squared Euclidean norm of a scattering vector $S x$ is the sum of its coefficients squared at all orders:

$$
\begin{aligned}
\|S x\|^{2} & =\sum_{m=0}^{l}\left\|S_{m} x\right\|^{2} \\
& =\sum_{m=0}^{l} \sum_{\lambda_{1}, \ldots, \lambda_{m}} \int\left|S_{m} x\left(t, \lambda_{1}, \ldots, \lambda_{m}\right)\right|^{2} d t .
\end{aligned}
$$

We consider deformations $x_{\tau}(t)=x(t-\tau(t))$ with $\left|\tau^{\prime}(t)\right|<1$ and $\sup _{t}|\tau(t)| \ll T$, which means that the maximum displacement is small relatively to the support of $\phi$. One can 


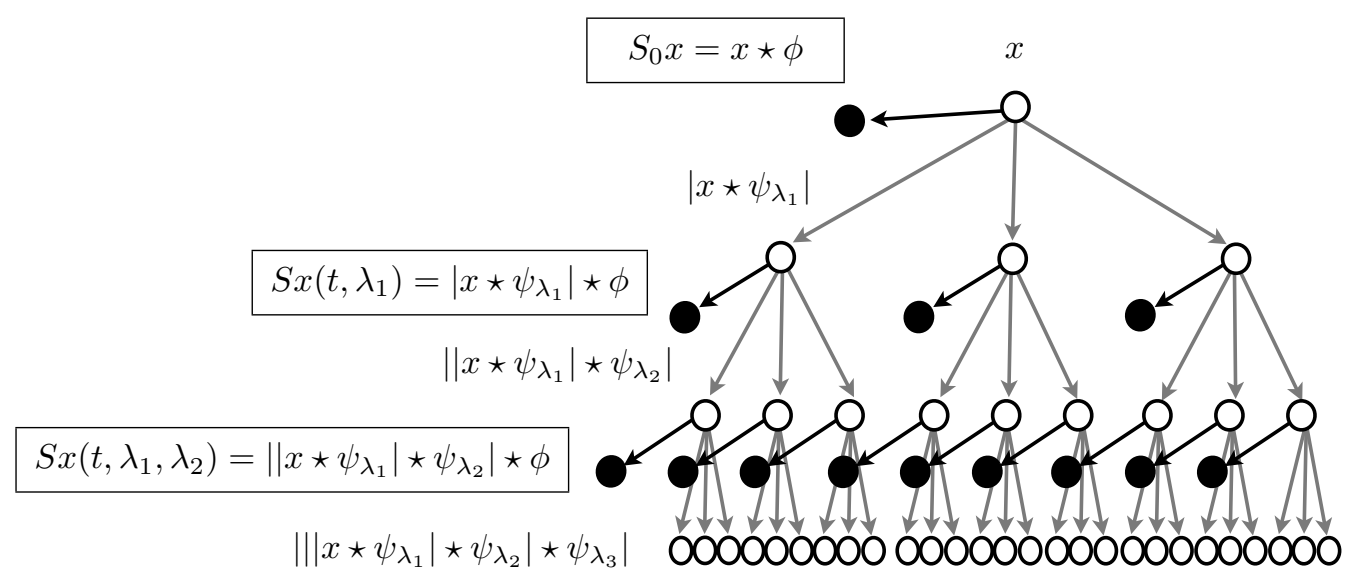

Fig. 4. A scattering transform iterates on wavelet modulus operators $\left|W_{m}\right|$ to compute cascades of $m$ wavelet convolutions and moduli stored in $U_{m} x$, and to output averaged scattering coefficients $S_{m} x$.

prove that there exists a constant $C$ such that for all $x$ and any such $\tau[11]$ :

$$
\left\|S x_{\tau}-S x\right\| \leq C \sup _{t}\left|\tau^{\prime}(t)\right|\|x\|,
$$

up to second-order terms. As explained for mel-spectral decompositions, the constant $C$ is inversely proportional to the octave bandwidth of wavelet filters. Over multiple scattering layers, we get $C=C_{0}\left(\max _{m} Q_{m}\right)$. For Morlet wavelets, numerical experiments on many examples give $C_{0} \approx 2$.

\section{B. Contraction and Energy Conservation}

We show that a scattering transform is contractive and can preserve energy. We denote $\|A x\|^{2}$ the squared Euclidean norm of a vector of coefficients $A x$, such as $W_{m} x, S_{m} x, U_{m} x$ or $S x$. Since $S x$ is computed by cascading wavelet modulus operators $\left|W_{m}\right|$, which are all contractive, it results that $S$ is also contractive:

$$
\left\|S x-S x^{\prime}\right\| \leq\left\|x-x^{\prime}\right\| .
$$

A scattering transform is therefore stable to additive noise.

If each wavelet transform is a tight frame, that is $\alpha=0$ in (15), each $\left|W_{m}\right|$ preserves the signal norm. Applying this property to $\left|W_{m+1}\right| U_{m} x=\left(S_{m} x, U_{m+1} x\right)$ yields

$$
\left\|U_{m} x\right\|^{2}=\left\|S_{m} x\right\|^{2}+\left\|U_{m+1} x\right\|^{2} .
$$

Summing these equations $0 \leq m \leq l$ proves that

$$
\|x\|^{2}=\|S x\|^{2}+\left\|U_{l+1} x\right\|^{2} .
$$

Under appropriate assumptions on the mother wavelet $\psi$, one can prove that $\left\|U_{l+1} x\right\|$ goes to zero as $l$ increases, which implies that $\|S x\|=\|x\|$ for $l=\infty[11]$. This property comes from the fact that the modulus of analytic wavelet coefficients computes a smooth envelope, and hence pushes energy towards lower frequencies. By iterating on wavelet modulus operators, the scattering transform progressively propagates all the energy of $U_{m} x$ towards lower frequencies, which is captured by the low-pass filter of scattering coefficients $S_{m} x=U_{m} x \star \phi$.

One can verify numerically that $\left\|U_{l+1} x\right\|$ converges to zero exponentially when $l$ goes to infinity and hence that $\|S x\|$

\begin{tabular}{|c|cccc|}
\hline $\mathrm{T}$ & $m=0$ & $m=1$ & $m=2$ & $m=3$ \\
\hline $23 \mathrm{~ms}$ & $0.0 \%$ & $94.5 \%$ & $4.8 \%$ & $0.2 \%$ \\
$93 \mathrm{~ms}$ & $0.0 \%$ & $68.0 \%$ & $29.0 \%$ & $1.9 \%$ \\
$370 \mathrm{~ms}$ & $0.0 \%$ & $34.9 \%$ & $53.3 \%$ & $11.6 \%$ \\
$1.5 \mathrm{~s}$ & $0.0 \%$ & $27.7 \%$ & $56.1 \%$ & $24.7 \%$ \\
\hline
\end{tabular}

\section{TABLE I}

Averaged Values $\left\|S_{m} x\right\|^{2} /\|x\|^{2}$ COMPUted for Signals $x$ IN THE TIMIT SPEECH DATASET [33], AS A FUNCTION OF ORDER $m$ AND AVERAGING SCALE $T$. FOR $m=1, S_{m} x$ IS CALCULATED By MORLET WAVELETS WITH $Q_{1}=8$, AND FOR $m=2,3$ BY CUBIC SPLINE WAVELETS WITH $Q_{2}=Q_{3}=1$.

converges exponentially to $\|x\|$. Table I gives the fraction of energy $\left\|S_{m} x\right\|^{2} /\|x\|^{2}$ absorbed by each scattering order. Since audio signals have little energy at low frequencies, $S_{0} x$ is very small and most of the energy is absorbed by $S_{1} x$ for $T=23 \mathrm{~ms}$. This explains why mel-frequency spectrograms are typically sufficient at these small time scales. However, as $T$ increases, a progressively larger proportion of energy is absorbed by higher-order scattering coefficients. For $T=1.5 \mathrm{~s}$, about $56 \%$ of the signal energy is captured in $S_{2} x$. Section VI shows that at this time scale, important amplitude modulation information is carried by these secondorder coefficients. For $T=1.5 \mathrm{~s}, S_{3} x$ carries $25 \%$ of the signal energy. It increases as $T$ increases, but for audio classification applications studied in this paper, $T$ remains below $1.5 \mathrm{~s}$, so these third-order coefficients are less important than first- and second-order coefficients. We therefore concentrate on secondorder scattering representations:

$$
S x=\left(S_{0} x(t), S_{1} x\left(t, \lambda_{1}\right), S_{2} x\left(t, \lambda_{1}, \lambda_{2}\right)\right)_{t, \lambda_{1}, \lambda_{2}} .
$$

\section{Fast Scattering Computation}

Subsampling scattering vectors provide a reduced representation, which leads to a faster implementation. Since the averaging window $\phi$ has a duration of the order of $T$, we compute scattering vectors with half-overlapping windows at $t=k T / 2$ with $k \in \mathbb{Z}$. 
We suppose that $x(t)$ has $N$ samples over each frame of duration $T$, and is thus sampled at a rate $N / T$. For each time frame $t=k T / 2$, the number of first-order wavelets $\psi_{\lambda_{1}}$ is about $Q_{1} \log _{2} N$ so there are about $Q_{1} \log _{2} N$ firstorder coefficients $S_{1} x\left(t, \lambda_{1}\right)$. We now show that the number of non-negligible second-order coefficients $S_{2} x\left(t, \lambda_{1}, \lambda_{2}\right)$ which needs to be computed is about $Q_{1} Q_{2}\left(\log _{2} N\right)^{2} / 2$.

The wavelet transform envelope $\left|x \star \psi_{\lambda_{1}}(t)\right|$ is a demodulated signal having approximately the same frequency bandwidth as $\widehat{\psi}_{\lambda_{1}}$. Its Fourier transform is mostly supported in the interval $\left[-\lambda_{1} Q_{1}^{-1}, \lambda_{1} Q_{1}^{-1}\right]$ for $\lambda_{1} \geq 2 \pi Q_{1} / T$, and in $\left[-2 \pi T^{-1}, 2 \pi T^{-1}\right]$ for $\lambda_{1} \leq 2 \pi Q_{1} / T$. If the support of $\widehat{\psi}_{\lambda_{2}}$ centered at $\lambda_{2}$ does not intersect the frequency support of $\left|x \star \psi_{\lambda_{1}}\right|$, then

$$
|| x \star \psi_{\lambda_{1}}\left|\star \psi_{\lambda_{2}}\right| \approx 0 .
$$

One can verify that non-negligible second-order coefficients satisfy

$$
\lambda_{2} \leq \max \left(\lambda_{1} Q_{1}^{-1}, 2 \pi T^{-1}\right) .
$$

For a fixed $t$, a direct calculation then shows that there are of the order of $Q_{1} Q_{2}\left(\log _{2} N\right)^{2} / 2$ second-order scattering coefficients. Similar reasoning extends this result to show that there are about $Q_{1} \ldots Q_{m}\left(\log _{2} N\right)^{m} / m$ ! non-negligible $m$ thorder scattering coefficients.

To compute $S_{1} x$ and $S_{2} x$ we first calculate $U_{1} x$ and $U_{2} x$ and average them with $\phi$. Over a time frame of duration $T$, to reduce computations while avoiding aliasing, $\left|x \star \psi_{\lambda_{1}}(t)\right|$ is subsampled at a rate which is twice its bandwidth. The family of filters $\left\{\widehat{\psi}_{\lambda_{1}}\right\}_{\lambda_{1} \in \Lambda_{1}}$ covers the whole frequency domain and $\Lambda_{1}$ is chosen so that filter supports barely overlap. Over a time frame where $x$ has $N$ samples, with the above subsampling we compute approximately $2 N$ first-order wavelet coefficients $\left\{\left|x \star \psi_{\lambda_{1}}(t)\right|\right\}_{t, \lambda_{1} \in \Lambda_{1}}$. Similarly, ||$x \star \psi_{\lambda_{1}}\left|\star \psi_{\lambda_{2}}(t)\right|$ is subsampled in time at a rate twice its bandwidth. Over the same time frame, the total number of second-order wavelet coefficients for all $t, \lambda_{1}$ and $\lambda_{2}$ stays below $2 N$. With a fast Fourier transform (FFT), these first- and second-order wavelet modulus coefficients are computed using $O(N \log N)$ operations. The resulting scattering coefficients $S_{1} x\left(t, \lambda_{1}\right)$ and $S_{2} x\left(t, \lambda_{1}, \lambda_{2}\right)$ are also calculated with $O(N \log N)$ operations, with FFT convolutions with $\phi$.

\section{INVERSE SCATTERING}

To better understand the information carried by scattering coefficients, this section studies a numerical inversion of the transform. Since a scattering transform is computed by cascading wavelet modulus operators $\left|W_{m}\right|$, the inversion approximately inverts each $\left|W_{m}\right|$ for $m<l$. At the maximum depth $m=l$, the algorithm begins with a deconvolution, estimating $U_{l} x(t)$ at all $t$ on the sampling grid of $x(t)$, from $S_{l} x(k T / 2)=U_{l} x \star \phi(k T / 2)$.

Because of the subsampling, one cannot compute $U_{l} x$ from $S_{l} x$ exactly. This deconvolution is thus the main source of error. To take advantage of the fact that $U_{l} x \geq 0$, the deconvolution is computed with the Richardson-Lucy algorithm [34], which preserves positivity if $\phi \geq 0$. We initialize $y_{0}(t)$ by interpolating $S_{l} x(k T / 2)$ linearly on the sampling grid of $x$, which introduces error because of aliasing. The RichardsonLucy deconvolution iteratively computes

$$
y_{n+1}(t)=y_{n}(t) \cdot\left[\left(\frac{y_{0}}{y_{n} \star \phi}\right) \star \widetilde{\phi}(t)\right],
$$

with $\widetilde{\phi}(t)=\phi(-t)$. Since it converges to the pseudo-inverse of the convolution operator applied to $y_{0}$, it blows up when $n$ increases because of the deconvolution instability. Deconvolution algorithms thus stop the iterations after a fixed number of iterations, which is set to 30 in this application. The result is then our estimate of $U_{l} x$.

Once an estimation of $U_{l} x$ is calculated by deconvolution, we compute an estimate $\widetilde{x}$ of $x$ by inverting each $\left|W_{m}\right|$ for $l \geq m>0$. The wavelet transform of a signal $x$ of size $N$ is a vector $W x=\left(x \star \phi, x \star \psi_{\lambda}\right)_{\lambda \in \Lambda}$ of about $Q N \log _{2} N$ coefficients, where $Q$ is the number of wavelets $\psi_{\lambda}$ per octave. These coefficients live in a subspace $\mathbf{V}$ of dimension $N$. To recover $W x$ from $|W| x=\left(x \star \phi,\left|x \star \psi_{\lambda}\right|\right)_{\lambda \in \Lambda}$, we search for a vector in $\mathbf{V}$ whose modulus values are specified by $|W| x$. This a non-convex optimization problem. Recent convex relaxation approaches [35], [36] are able to compute exact solutions, but they require too much computation and memory for audio applications. Since the main source of errors is introduced at the deconvolution stage, one can use an approximate but fast inversion algorithm. The inversion of $|W|$ is typically more stable when $|W| x$ is sparse because there is no phase to recover if $\left|x \star \psi_{\lambda}\right|=0$. This motivates using wavelets $\psi_{\lambda_{m}}$ which provide sparse representations at each order $m$.

Griffin \& Lim [37] showed that alternating projections recovers good quality audio signals from spectrogram values, but with large mean-square errors because the algorithm is trapped in local minima. The same algorithm inverts $|W|$ by alternating projections on the wavelet transform space $\mathbf{V}$ and on the modulus constraints. An estimation $\widetilde{x}$ of $x$ is calculated from $|W| x$, by initializing $\widetilde{x}_{0}$ to be a Gaussian white noise. For any $n \geq 0, \widetilde{x}_{n+1}$ is computed from $\widetilde{x}_{n}$ by first adjusting the modulus of its wavelet coefficients, with a non-linear projector

$$
z_{\lambda}(t)=\left|x \star \psi_{\lambda}(t)\right| \frac{\widetilde{x}_{n} \star \psi_{\lambda}(t)}{\left|\widetilde{x}_{n} \star \psi_{\lambda}(t)\right|} .
$$

Applying the wavelet transform pseudo-inverse (17) yields

$$
\widetilde{x}_{n+1}=x \star \phi \star \bar{\phi}(t)+\sum_{\lambda \in \Lambda} \operatorname{Real}\left(z_{\lambda} \star \bar{\psi}_{\lambda}(t)\right) .
$$

The dual filters are defined in (18). One can verify that $W \widetilde{x}_{n+1}$ is the orthogonal projection of $\left\{x \star \phi, z_{\lambda}\right\}_{\lambda \in \Lambda}$ in $\mathbf{V}$. Numerical experiments are performed with $n=30$ iterations, and we set $\widetilde{x}=\widetilde{x}_{n}$.

When $l=1$, an approximation $\widetilde{x}$ of $x$ is computed from from $\left(S_{0} x, S_{1} x\right)$ by first estimating $U_{1} x$ from $S_{1} x=U_{1} x \star \phi$ with the Richardson-Lucy deconvolution algorithm. We then compute $\widetilde{x}$ from $S_{0} x$ and this estimation of $U_{1} x$ by approximately inverting $\left|W_{1}\right|$ with the Griffin \& Lim algorithm. When $T$ is above $100 \mathrm{~ms}$, the deconvolution loses too much information, and audio reconstructions obtained from firstorder coefficients are crude. Figure 5 (a) shows the scalograms $\log \left|x \star \psi_{\lambda_{1}}(t)\right|$ of a speech and a music signal, and the 
$\log \lambda_{1}$

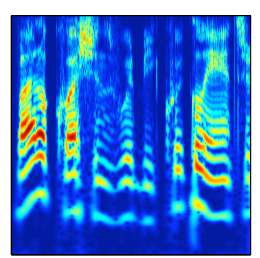

$\log \lambda_{1}$

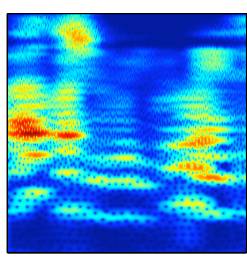

$\log \lambda_{1}$

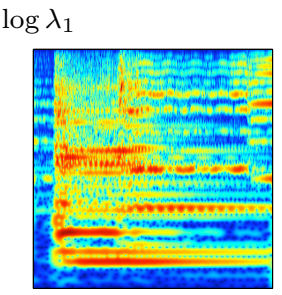

(a)

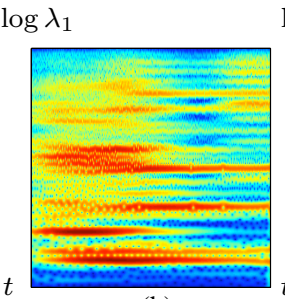

(b)

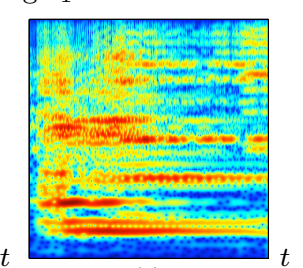

(c)

Fig. 5. (a): Scalogram $\log \left|x \star \psi_{\lambda_{1}}(t)\right|$ for recordings of speech (top) and a cello (bottom). $(b, c)$ : Scalograms $\log \left|\widetilde{x} \star \psi_{\lambda_{1}}(t)\right|$ of reconstructions $\widetilde{x}$ from first-order scattering coefficients $(l=1)$ in $(b)$, and from first- and secondorder coefficients $(l=2)$ in $(c)$. Scattering coefficients were computed with $T=190 \mathrm{~ms}$ for the speech signal and $T=370 \mathrm{~ms}$ for the cello signal.

scalograms $\log \left|\widetilde{x} \star \psi_{\lambda_{1}}(t)\right|$ of their approximations $\widetilde{x}$ from first-order scattering coefficients.

When $l=2$, the approximation $\widetilde{x}$ is calculated from $\left(S_{0} x, S_{1} x, S_{2} x\right)$ by applying the deconvolution algorithm to $S_{2} x=U_{2} x \star \phi$ to estimate $U_{2} x$, and then by successively inverting $\left|W_{2}\right|$ and $\left|W_{1}\right|$ with the Griffin \& Lim algorithm. Figure 5(c) shows $\log \left|\widetilde{x} \star \psi_{\lambda_{1}}(t)\right|$ for the same speech and music signals. Amplitude modulations, vibratos and attacks are restored with greater precision by incorporating second-order coefficients, yielding much better audio quality compared to first-order reconstructions. However, even with $l=2$, reconstructions become crude for $T \geq 500 \mathrm{~ms}$. Indeed, the number of second-order scattering coefficients $Q_{1} Q_{2} \log _{2}^{2} N / 2$ is too small relatively to the number $N$ audio samples in each audio frame, and they do not capture enough information. Examples of audio reconstructions are available at http://www.di.ens.fr/data/scattering/audio/

\section{Normalized SCATTERING SPECTRUM}

To reduce redundancy and increase invariance, Section VI-A normalizes scattering coefficients. Section VI-B shows that normalized second-order coefficients provide high-resolution spectral information through interferences. Section VI-C also proves that they characterize amplitude modulations of audio signals.

\section{A. Normalized Scattering Transform}

Scattering coefficients are renormalized to increase their invariance. It also decorrelates these coefficients at different orders. First-order scattering coefficients are renormalized so that they become insensitive to multiplicative constants:

$$
\widetilde{S}_{1} x\left(t, \lambda_{1}\right)=\frac{S_{1} x\left(t, \lambda_{1}\right)}{|x| \star \phi(t)+\epsilon} .
$$

The constant $\epsilon$ is a silence detection threshold so that $\widetilde{S}_{1} x=0$ if $x=0$, which may be set to 0 .
The lowpass filter $\phi(t)$ can be wider than the one used in the scattering transform. Specifically, if we want to retain local amplitude information of $S_{1} x$ below a certain scale, we can normalize by the average of $|x|$ over this scale, creating invariance only to amplitude changes over larger intervals.

At any order $m \geq 2$, scattering coefficients are renormalized by coefficients of the previous order:

$$
\widetilde{S}_{m} x\left(t, \lambda_{1}, \ldots, \lambda_{m-1}, \lambda_{m}\right)=\frac{S_{m} x\left(t, \lambda_{1}, \ldots, \lambda_{m-1}, \lambda_{m}\right)}{S_{m-1} x\left(t, \lambda_{1}, \ldots, \lambda_{m-1}\right)+\epsilon} .
$$

A normalized scattering representation is defined by $\widetilde{S} x=$ $\left(\widetilde{S}_{m} x\right)_{1 \leq m \leq l}$. We shall mostly limit ourselves to $l=2$.

For $m=2$,

$$
\widetilde{S}_{2} x\left(t, \lambda_{1}, \lambda_{2}\right)=\frac{S_{2} x\left(t, \lambda_{1}, \lambda_{2}\right)}{S_{1} x\left(t, \lambda_{1}\right)+\epsilon} .
$$

Let us show that these coefficients are nearly invariant to a filtering by $h(t)$ if $\widehat{h}(\omega)$ is approximately constant on the support of $\widehat{\psi}_{\lambda_{1}}$. This condition is satisfied if

$$
\frac{\lambda_{1}}{Q_{1}} \ll\left(\int|t||h(t)| d t\right)^{-1}
$$

It implies that $h \star \psi_{\lambda_{1}}(t) \approx \widehat{h}\left(\lambda_{1}\right) \psi_{\lambda_{1}}(t)$, and hence $\mid(x \star h) \star$ $\psi_{\lambda_{1}}(t)|\approx| \widehat{h}\left(\lambda_{1}\right)|| x \star \psi_{\lambda_{1}}(t) \mid$. It results that

$$
S_{1}(x \star h)\left(t, \lambda_{1}\right) \approx\left|\widehat{h}\left(\lambda_{1}\right)\right| S_{1} x\left(t, \lambda_{1}\right) .
$$

Similarly, $S_{2}(x \star h)\left(t, \lambda_{1}, \lambda_{2}\right) \approx\left|\widehat{h}\left(\lambda_{1}\right)\right| S_{2} x\left(t, \lambda_{1}, \lambda_{2}\right)$, so after normalization

$$
\widetilde{S}_{2}(x \star h)\left(t, \lambda_{1}, \lambda_{2}\right) \approx \widetilde{S}_{2} x\left(t, \lambda_{1}, \lambda_{2}\right) .
$$

Normalized second-order coefficients are thus invariant to filtering by $h(t)$. One can verify that this remains valid at any order $m \geq 2$.

\section{B. Frequency Interval Measurement from Interference}

A wavelet transform has a worse frequency resolution than a windowed Fourier transform at high frequencies. However, we show that frequency intervals between harmonics are accurately measured by second-order scattering coefficients.

Suppose $x$ has two frequency components in the support of $\widehat{\psi}_{\lambda_{1}}$. We then have

$$
x \star \psi_{\lambda_{1}}(t)=\alpha_{1} e^{i \xi_{1} t}+\alpha_{2} e^{i \xi_{2} t},
$$

whose modulus squared equals

$$
\left|x \star \psi_{\lambda_{1}}(t)\right|^{2}=\left|\alpha_{1}\right|^{2}+\left|\alpha_{2}\right|^{2}+2\left|\alpha_{1} \alpha_{2}\right| \cos \left(\xi_{1}-\xi_{2}\right) t .
$$

We approximate $\left|x \star \psi_{\lambda_{1}}(t)\right|$ with a first-order expansion of the square root, which yields

$$
\left|x \star \psi_{\lambda_{1}}(t)\right| \approx \sqrt{\left|\alpha_{1}\right|^{2}+\left|\alpha_{2}\right|^{2}}+\frac{\left|\alpha_{1} \alpha_{2}\right|}{\sqrt{\left|\alpha_{1}\right|^{2}+\left|\alpha_{2}\right|^{2}}} \cos \left(\xi_{1}-\xi_{2}\right) t .
$$

If $\phi$ has a support of size $T \gg\left|\xi_{1}-\xi_{2}\right|^{-1}$, then $S_{1} x\left(t, \lambda_{1}\right) \approx$ $\sqrt{\left|\alpha_{1}\right|^{2}+\left|\alpha_{2}\right|^{2}}$, so $\widetilde{S}_{2} x\left(t, \lambda_{1}, \lambda_{2}\right)=\frac{S_{2} x\left(t, \lambda_{1}, \lambda_{2}\right)}{S_{1} x\left(t, \lambda_{1}\right)+\epsilon}$ satisfies

$$
\widetilde{S}_{2} x\left(t, \lambda_{1}, \lambda_{2}\right) \approx\left|\widehat{\psi}_{\lambda_{2}}\left(\xi_{2}-\xi_{1}\right)\right| \frac{\left|\alpha_{1} \alpha_{2}\right|}{\left|\alpha_{1}\right|^{2}+\left|\alpha_{2}\right|^{2}} .
$$


These normalized second-order coefficients are thus nonnegligible when $\lambda_{2}$ is of the order of the frequency interval $\left|\xi_{2}-\xi_{1}\right|$. This shows that although the first wavelet $\widehat{\psi}_{\lambda_{1}}$ does not have enough resolution to discriminate the frequencies $\xi_{1}$ and $\xi_{2}$, second-order coefficients detect their presence and accurately measure the interval $\left|\xi_{2}-\xi_{1}\right|$. As in audio perception, scattering coefficients can accurately measure frequency intervals but not frequency location. The normalized secondorder scattering coefficients (38) are large only if $\alpha_{1}$ and $\alpha_{2}$ have the same order of magnitude. This also conforms to auditory perception where a frequency interval is perceived only when the two frequency components have a comparable amplitude.

If $x \star \psi_{\lambda_{1}}(t)=\sum_{n} \alpha_{n} e^{i \xi_{n} t}$ has more frequency components, we verify similarly that $\widetilde{S}_{2} x\left(t, \lambda_{1}, \lambda_{2}\right)$ is non-negligible when $\lambda_{2}$ is of the order of $\left|\xi_{n}-\xi_{n^{\prime}}\right|$ for some $n \neq n^{\prime}$. These coefficients can thus measure multiple frequency intervals within the frequency band covered by $\widehat{\psi}_{\lambda_{1}}$. If the frequency resolution of $\widehat{\psi}_{\lambda_{2}}$ is not sufficient to discriminate between two frequency intervals $\left|\xi_{1}-\xi_{2}\right|$ and $\left|\xi_{3}-\xi_{4}\right|$, these intervals will interfere and create high amplitude third-order scattering coefficients. A similar calculation shows that third-order scattering coefficients $\widetilde{S}_{3} x\left(t, \lambda_{1}, \lambda_{2}, \lambda_{3}\right)$ detect the presence of two such intervals within the support of $\widehat{\psi}_{\lambda_{2}}$ when $\lambda_{3}$ is close to ||$\xi_{1}-\xi_{2}|-| \xi_{3}-\xi_{4}||$. They thus measure "intervals of intervals."

Figure 6(a) shows the scalogram $\log \left|x \star \psi_{\lambda_{1}}\right|$ of a signal $x$ containing a chord with two notes, whose fundamental frequencies are $\xi_{1}=600 \mathrm{~Hz}$ and $\xi_{2}=675 \mathrm{~Hz}$, followed by an arpeggio of the same two notes. First-order coefficients $\log \widetilde{S}_{1} x\left(t, \lambda_{1}\right)$ in Figure 6(b) are very similar for the chord and the arpeggio because the time averaging loses time localization. However they are easily differentiated in Figure 6(c), which displays $\log \widetilde{S}_{2} x\left(t, \lambda_{1}, \lambda_{2}\right)$ for $\lambda_{1} \approx \xi_{1}=600 \mathrm{~Hz}$, as a function of $\lambda_{2}$. The chord creates large amplitude coefficients for $\lambda_{2}=\left|\xi_{2}-\xi_{1}\right|=75 \mathrm{~Hz}$, which disappear for the arpeggio because these two frequencies are not present simultaneously. Second-order coefficients have also a large amplitude at low frequencies $\lambda_{2}$. These arise from variation of the note envelopes in the chord and in the arpeggio, as explained in the next section.

\section{Amplitude Modulation Spectrum}

Audio signals are usually modulated in amplitude by an envelope, whose variations may correspond to an attack or a tremolo. For voiced and unvoiced sounds, we show that amplitude modulations are characterized by normalized secondorder scattering coefficients.

Let $x(t)$ be a sound resulting from an excitation $e(t)$ filtered by a resonance cavity of impulse response $h(t)$, which is modulated in amplitude by $a(t) \geq 0$ to give

$$
x(t)=a(t)(e \star h)(t) .
$$

We shall start by taking $e$ to be a pulse train of pitch $\xi$ given by

$$
e(t)=\frac{2 \pi}{\xi} \sum_{n} \delta\left(t-\frac{2 n \pi}{\xi}\right)=\sum_{k} e^{i k \xi t},
$$

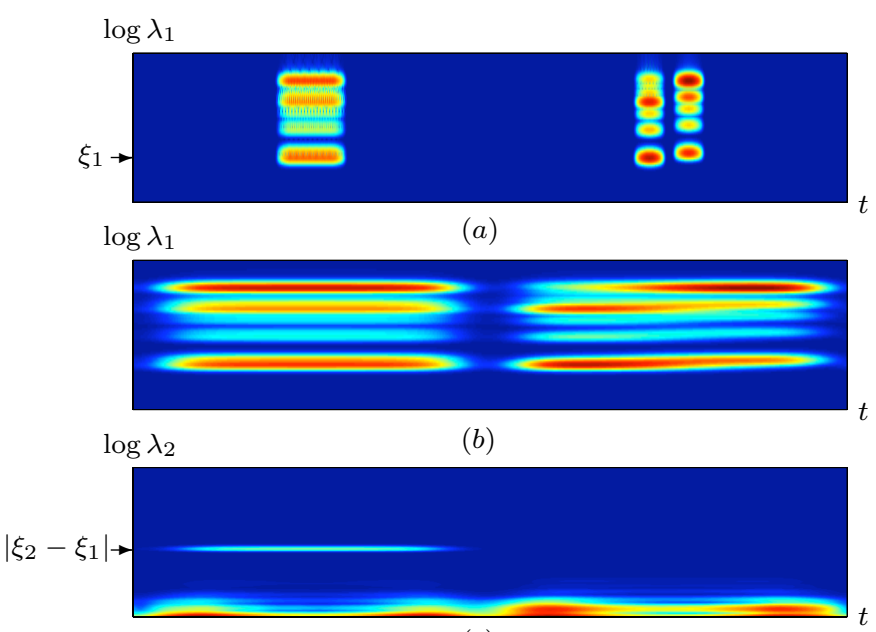

(c)

Fig. 6. (a): Scalogram $\log \left|x \star \psi_{\lambda_{1}}(t)\right|$ for a signal with two notes, of fundamental frequencies $\xi_{1}=600 \mathrm{~Hz}$ and $\xi_{2}=675 \mathrm{~Hz}$, first played as a chord and then as an arpeggio. (b): First-order normalized scattering coefficients $\log \widetilde{S}_{1} x\left(t, \lambda_{1}\right)$ for $T=512 \mathrm{~ms}$. (c): Second-order normalized scattering coefficients $\log \widetilde{S}_{2}\left(t, \xi_{1}, \lambda_{2}\right)$ with $\lambda_{1}=\xi_{1}$ as a function of $t$ and $\lambda_{2}$. The chord interferences produce large coefficients for $\lambda_{2}=\left|\xi_{2}-\xi_{1}\right|$.

representing a voiced sound. The impulse response $h(t)$ is typically very short compared to the minimum variation interval $\left(\sup _{t}\left|a^{\prime}(t)\right|\right)^{-1}$ of the modulation term and is smaller than $2 \pi / \xi$.

We consider $\psi_{\lambda_{1}}$ whose time support is short relatively to $\left(\sup _{t}\left|a^{\prime}(t)\right|\right)^{-1}$ and to the averaging interval $T$, and whose frequency bandwidth is smaller than the pitch $\xi$ and to the minimum variation interval of $\hat{h}$. These conditions are satisfied if

$$
\left(\int|t||h(t)| d t\right)^{-1} \gg \frac{\lambda_{1}}{Q_{1}} \gg \sup _{t}\left|a^{\prime}(t)\right|,
$$

After normalization $\widetilde{S}_{1} x\left(t, \lambda_{1}\right)=\frac{S_{1} x\left(t, \lambda_{1}\right)}{|x| \star \phi(t)+\epsilon}$, Appendix A shows that

$$
\widetilde{S}_{1} x\left(t, \lambda_{1}\right) \approx\left|\widehat{\psi}_{\lambda_{1}}(k \xi)\right| \frac{\left|\widehat{h}\left(\lambda_{1}\right)\right|}{\|h\|_{1}}
$$

where $\|h\|_{1}(t)=\int|h(t)| d t$ and $k$ is an integer such that $\mid k \xi-$ $\lambda_{1} \mid<\xi / 2$. First-order coefficients are thus proportional to the spectral envelope $\left|\widehat{h}\left(\lambda_{1}\right)\right|$ if $\lambda_{1} \approx k \xi$ is close to a harmonic frequency.

Similarly, for $\widetilde{S}_{2} x\left(t, \lambda_{1}, \lambda_{2}\right)=\frac{S_{2} x\left(t, \lambda_{1}, \lambda_{2}\right)}{S_{1} x\left(t, \lambda_{1}\right)+\epsilon}$, Appendix A shows that

$$
\widetilde{S}_{2} x\left(t, \lambda_{1}, \lambda_{2}\right) \approx \frac{\left|a \star \psi_{\lambda_{2}}\right| \star \phi(t)}{a \star \phi(t)} .
$$

Second-order coefficients thus do not depend upon $h$ and $\xi$ but only on the amplitude modulation $a(t)$ provided that $S_{1} x\left(t, \lambda_{1}\right)$ is non-negligible.

Figure 7 (a) displays $\log \left|x \star \psi_{\lambda_{1}}(t)\right|$ for a signal having three voiced and three unvoiced sounds. The first three are produced by a pulse train excitation $e(t)$ with a pitch of $\xi=600 \mathrm{~Hz}$. Figure 7 (b) shows that $\log \widetilde{S}_{1} x\left(t, \lambda_{1}\right)$ has a harmonic structure, with an amplitude depending on $\log \left|\widehat{h}\left(\lambda_{1}\right)\right|$. The averaging 


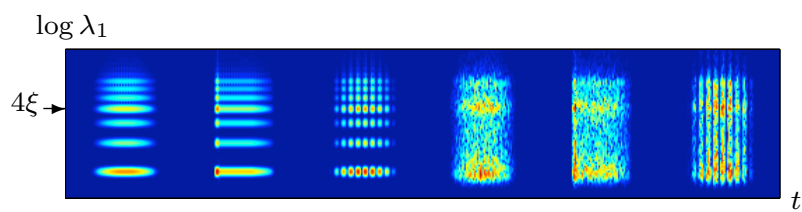

$\log \lambda_{1}$

$(a)$

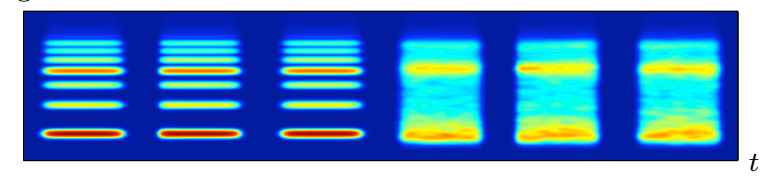

$\log \lambda_{2}$

(b)

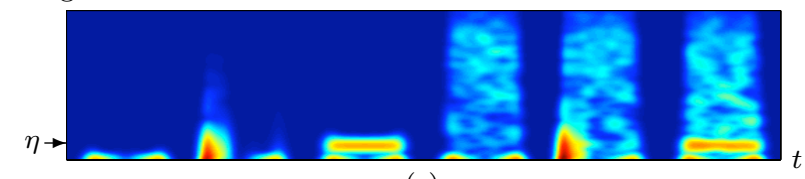

(c)

Fig. 7. (a): Scalogram $\log \left|x \star \psi_{\lambda_{1}}(t)\right|$ for a signal with three voiced sounds of same pitch $\xi=600 \mathrm{~Hz}$ and same $h(t)$ but different amplitude modulations $a(t)$ : first a smooth attack, then a sharp attack, then a tremolo of frequency $\eta$. It is followed by three unvoiced sounds created with the same $h(t)$ and same amplitude modulations $a(t)$ as the first three voiced sounds. $(b)$ : First-order scattering $\log \widetilde{S}_{1} x\left(t, \lambda_{1}\right)$ with $T=128 \mathrm{~ms}$. (c): Second-order scattering $\log \widetilde{S}_{2} x\left(t, \lambda_{1}, \lambda_{2}\right)$ displayed for $\lambda_{1}=4 \xi$, as a function of $t$ and $\lambda_{2}$.

by $\phi$ and the normalization remove the effect of the different modulation amplitudes $a(t)$ of these three voiced sounds.

Figure 7 (c) displays $\log \widetilde{S}_{2}\left(t, \lambda_{1}, \lambda_{2}\right)$ for the fourth partial $\lambda_{1}=4 \xi$, as a function of $\lambda_{2}$. The modulation envelope $a(t)$ of the first sound has a smooth attack and thus produces large coefficients only at low frequencies $\lambda_{2}$. The envelope $a(t)$ of the second sound has a much sharper attack and thus produces large amplitude coefficients for higher frequencies $\lambda_{2}$. The third sound is modulated by a tremolo, which is a periodic oscillation $a(t)=1+\epsilon \cos (\eta t)$. According to 43, this tremolo creates large amplitude coefficients when $\lambda_{2}=\eta$, as shown in Figure 7(c).

Unvoiced sounds are modeled by excitations $e(t)$ which are realizations of Gaussian white noise. The modulation amplitude is typically non-sparse, which means the square of the average of $a(t)$ on intervals of size $T$ is of the order of the average of $a^{2}(t)$. Appendix $\mathrm{A}$ shows that

$$
\widetilde{S}_{1} x\left(t, \lambda_{1}\right) \approx \frac{\pi\|\psi\|}{2^{3 / 2}} \lambda_{1}{ }^{1 / 2} \frac{\left|\widehat{h}\left(\lambda_{1}\right)\right|}{\|h\|} .
$$

Similarly to 42$), \widetilde{S}_{1} x\left(t, \lambda_{1}\right)$ is proportional to $\left|\widehat{h}\left(\lambda_{1}\right)\right|$ but does not have a harmonic structure. This is shown in Figure 7.b) by the last three unvoiced sounds. The fourth, fifth, and sixth sounds have the same filter $h(t)$ and envelope $a(t)$ as the first, second, and third sounds, respectively, but with a Gaussian white noise excitation $e(t)$.

Similarly to 43, Appendix A also shows that

$$
\widetilde{S}_{2} x\left(t, \lambda_{1}, \lambda_{2}\right)=\frac{\left|a \star \psi_{\lambda_{2}}\right| \star \phi(t)}{a \star \phi(t)}+\widetilde{\epsilon}(t)
$$

where $\widetilde{\epsilon}(t)$ is small relatively to the first amplitude modulation term if $(4 / \pi-1)^{1 / 2}\left(\lambda_{2} Q_{1}\right)^{1 / 2}\left(\lambda_{1} Q_{2}\right)^{-1 / 2}$ is small relatively to this modulation term. For voiced and unvoiced sounds, $\widetilde{S}_{2} x\left(t, \lambda_{1}, \lambda_{2}\right)$ mainly depends upon the amplitude modulation $a(t)$. This is illustrated by Figure 7(c), which shows that the fourth, fifth, and sixth sounds have second-order coefficients similar to those of the first, second, and third sounds, respectively. The stochastic error term $\tilde{\epsilon}$ produced by unvoiced sounds appears as random low-amplitude fluctuations in Figure 7)(c).

\section{FREQUENCY TRANSPOSITION INVARIANCE}

Audio signals within the same class may be transposed in frequency. Frequency transposition occurs when a single word is pronounced by different speakers. It is a complex phenomenon which affects the pitch and the spectral envelope. The envelope is translated on a logarithmic frequency scale but also deformed. We thus need a representation which is invariant to frequency translation on a logarithmic scale, and which also is stable to frequency deformations. After reviewing the mel-frequency cepstral coefficient (MFCC) approach through the discrete cosine transform (DCT), this section defines such a representation with a scattering transform computed along log-frequency.

MFCCs are computed from the log-mel-frequency spectrogram $\log M x(t, \lambda)$ by calculating a DCT along the melfrequency index $\gamma$ for a fixed $t$ [38]. This $\gamma$ is linear in $\lambda$ for low frequencies, but is proportional to $\log _{2} \lambda$ for higher frequencies. For simplicity, we write $\gamma=\log _{2} \lambda$ and $\lambda=2^{\gamma}$, although this should be modified at low frequencies.

The frequency index of the DCT is called the "quefrency" parameter. In MFCCs, high-quefrency coefficients are often set to zero, which is equivalent to averaging $\log M x\left(t, 2^{\gamma}\right)$ along $\gamma$ and provides some frequency transposition invariance. The more high-quefrency coefficients are set to zero, the bigger the averaging and hence the more transposition invariance obtained, but at the expense of losing potentially important information.

The loss of information due to averaging along $\gamma$ can be recovered by computing wavelet coefficients along $\gamma$. We thus replace the DCT by a scattering transform along $\gamma$. A frequency scattering transform is calculated by iteratively applying wavelet transforms and modulus operators. An analytic wavelet transform of a log-frequency dependent signal $z(\gamma)$ is defined as in (13), but with convolutions along the logfrequency variable $\gamma$ instead of time:

$$
W^{\mathrm{fr}} z=\left(z \star \phi^{\mathrm{fr}}(\gamma), z \star \psi_{q}(\gamma)\right)_{\gamma, q} .
$$

Each wavelet $\psi_{q}$ is a band-pass filter whose Fourier transform $\widehat{\psi}_{q}$ is centered at "quefrency" $q$ and $\phi^{\mathrm{fr}}$ is an averaging filter. These wavelets satisfy the condition 15 , so $W^{\text {fr }}$ is contractive and invertible.

Although the scattering transform along $\gamma$ can be computed at any order, we restrict ourself to zero and first-order scattering coefficients, because it seems to be sufficient for classification. A first-order scattering transform of $z(\gamma)$ is calculated from

$$
U^{\mathrm{fr}} z=\left(z(\gamma),\left|z \star \psi_{q_{1}}(\gamma)\right|\right),
$$

by averaging these coefficients along $\gamma$ with $\phi^{\text {fr }}$ :

$$
S^{\mathrm{fr}} z=\left(z \star \phi^{\mathrm{fr}}(\gamma),\left|z \star \psi_{q_{1}}\right| \star \phi^{\mathrm{fr}}(\gamma)\right) .
$$


These coefficients are locally invariant to log-frequency shifts, over a domain proportional to the support of the averaging filter $\phi^{\mathrm{fr}}$. This frequency scattering is formally identical to a time scattering transform. It has the same properties if we replace the time $t$ by the log-frequency variable $\gamma$. Numerical experiments are implemented using Morlet wavelets $\psi_{q_{1}}$ with $Q_{1}=1$.

Similarly to MFCCs, we apply a logarithm to normalized scattering coefficients so that multiplicative components become additive and can be separated by linear operators. This was shown to improve classification performance. The logarithm of a second-order normalized time scattering, at a frequency $\lambda_{1}=2^{\gamma}$ and a time $t$ is

$$
\log \widetilde{S} x(t, \gamma)=\left(\begin{array}{l}
\log \widetilde{S}_{1} x\left(t, 2^{\gamma}\right) \\
\log \widetilde{S}_{2} x\left(t, 2^{\gamma}, \lambda_{2}\right)
\end{array}\right)_{\lambda_{2}}
$$

This is a vector of signals $z(\gamma)$, where $z$ depends on $t$ and $\lambda_{2}$. Let us transform each $z(\gamma)$ by the frequency scattering operators $U^{\mathrm{fr}}$ or $S^{\mathrm{fr}}$, defined in 46 and 47 . Let $U^{\mathrm{fr}} \log \widetilde{S} x(t, \gamma)$ and $S^{\mathrm{fr}} \log \widetilde{S} x(t, \gamma)$ stand for the concatenation of these transformed signals for all $t$ and $\lambda_{2}$. The representation $S^{\mathrm{fr}} \log \widetilde{S} x$ is calculated by cascading a scattering in time and a scattering in log-frequency. It is thus locally translation invariant in time and in log-frequency, and stable to time and frequency deformations. The interval of time-shift invariance is defined by the size of the time averaging window $\phi$, whereas its frequency-transposition invariance depends upon the width of the log-frequency averaging window $\phi^{\text {fr }}$.

Frequency transposition invariance is useful for certain tasks, such as speaker-independent speech recognition or transposition-independent melody recognition, but it removes information important to other tasks, such as speaker identification. The frequency transposition invariance, implemented by the frequency averaging filter $\phi^{\text {fr }}$, should thus be adapted to the classification task. Next section explains that this can be done by replacing $S^{\mathrm{fr}} \log \widetilde{S} x(t, \gamma)$ by $U^{\mathrm{fr}} \log \widetilde{S} x(t, \gamma)$ and optimizing the linear averaging at the supervised classification stage.

\section{Classification}

This section compares the classification performance of support vector machine classifiers applied to scattering representations with standard low-level features such as $\Delta$-MFCCs or more sophisticated state-of-the-art representations. Section VIII-A explains how to automatically adapt invariance parameters, while Sections VIII-B and VIII-C present results for musical genre classification and phone classification, respectively.

\section{A. Adapting Frequency Transposition Invariance}

The amount of frequency-transposition invariance depends on the classification problem, and may vary for each signal class. This adaptation is implemented by a supervised classifier, applied to the time and frequency scattering transform.

Figure 8 illustrates the computation of a time and frequency scattering representation. The normalized scattering transform

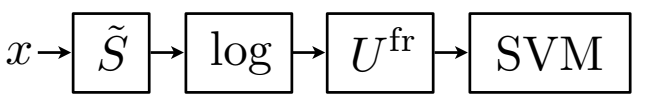

Fig. 8. A time and frequency scattering representation is computed by applying a normalized temporal scattering $\widetilde{S}$ on the input signal $x(t)$, a logarithm, and a scattering along log-frequency without averaging.

$\widetilde{S} x$ of an input signal $x$ is computed along time, over halfoverlapping windows of size $T$. The log-scattering vector for each time window is transformed along frequencies by the wavelet modulus operator $U^{\mathrm{fr}}$, as explained in Section VII Since we do not know in advance how much transposition invariance is needed for a particular classification task, the final frequency averaging is adaptively computed by the supervised classifier, which takes as input the vector of coefficients $\left\{U^{\mathrm{fr}} \log \widetilde{S} x(t, \gamma)\right\}_{\gamma}$, for each time frame indexed by $t$.

The supervised classification is implemented by a support vector machine (SVM). A binary SVM classifies a feature vector by calculating its position relative to a hyperplane, which is optimized to maximize class separation given a set of training samples. It thus computes the sign of an optimized linear combination of the feature vector coefficients. With a Gaussian kernel of variance $\sigma^{2}$, the SVM computes different hyperplanes in different balls of radius $\sigma$ in the feature space. The coefficients of the linear combination thus vary smoothly with the feature vector values. Applied to $\left\{U^{\text {fr }} \log S x(t, \gamma)\right\}_{\gamma}$, the SVM optimizes the linear combination of coefficients along $\gamma$, and can thus adjust the amount of linear averaging to create frequency-transposition invariant descriptors which maximize class separation. A multi-class SVM is computed from binary classifiers using a one-versus-one approach. All numerical experiments use the LIBSVM library [39].

The wavelet octave resolution $Q_{1}$ can also be adjusted at the supervised classification stage, by computing the time scattering for several values of $Q_{1}$ and concatenating all coefficients in a single feature vector. A filter bank with $Q_{1}=8$ has enough frequency resolution to separate harmonic structures, whereas wavelets with $Q_{1}=1$ have a smaller time support and can thus better localize transient in time. The linear combination optimized by the SVM is a feature selection algorithm, which can select the best coefficients to discriminate any two classes. In the experiments described below, adding more values of $Q_{1}$ between 1 and 8 provides marginal improvements.

\section{B. Musical Genre Classification}

Scattering feature vectors are first applied to musical genre classification problem on the GTZAN dataset [40]. The dataset consists of 1000 thirty-second clips, divided into 10 genres of 100 clips each. Given a clip, the goal is to find its genre.

Preliminary experiments have demonstrated the efficiency of the scattering transform for music classification [41] and for environmental sounds [42]. These results are improved by letting the supervised classifier adjust the transform parameters to the signal classes. A set of feature vectors is computed over half-overlapping frames of duration $T$. Each frame of a clip is classified separately by a Gaussian kernel SVM, and the 


\begin{tabular}{|c|c|c|}
\hline Representations & GTZAN & TIMIT \\
\hline$\Delta$-MFCC $(\mathrm{T}=23 \mathrm{~ms})$ & $20.2 \pm 5.4$ & 18.5 \\
\hline$\Delta$-MFCC $(\mathrm{T}=740 \mathrm{~ms})$ & $18.0 \pm 4.2$ & 60.5 \\
\hline State of the art (excluding scattering) & $9.4 \pm 3.1|8|$ & $16.7[43 \mid$ \\
\hline & $T=740 \mathrm{~ms}$ & $T=32 \mathrm{~ms}$ \\
\hline Time Scat., $l=1$ & $19.1 \pm 4.5$ & 19.0 \\
\hline Time Scat., $l=2$ & $10.7 \pm 3.1$ & 17.3 \\
\hline Time Scat., $l=3$ & $10.6 \pm 2.5$ & 18.1 \\
\hline Time \& Freq. Scat., $l=2$ & $9.3 \pm 2.4$ & 16.6 \\
\hline Adapt $Q_{1}$, Time \& Freq. Scat., $l=2$ & $8.6 \pm 2.2$ & 15.9 \\
\hline
\end{tabular}

TABLE II

ERROR RATES (IN PERCENT) FOR MUSICAL GENRE CLASSIFICATION ON GTZAN AND FOR PHONE CLASSIFICATION ON THE TIMIT DATABASE FOR DIFFERENT FEATURES. TIME SCATTERING TRANSFORMS ARE COMPUTED WITH $T=740 \mathrm{~ms}$ FOR GTZAN AND WITH $T=32 \mathrm{~ms}$ FOR TIMIT

clip is assigned to the class which is most often selected by its frames. To reduce the SVM training time, feature vectors were only computed every $370 \mathrm{~ms}$ for the training set. The SVM slack parameter and the Gaussian kernel variance are determined through cross-validation on the training data. Table II summarizes results with one run of ten-fold cross-validation. It gives the average error and its standard deviation.

Scattering classification results are first compared with results obtained with MFCC feature vectors. A $\triangle$-MFCC vector represents an audio frame of duration $T$ at time $t$ by three MFCC vectors centered at $t-T / 2, t$ and $t+T / 2$. When computed for $T=23 \mathrm{~ms}$, the $\Delta$-MFCC error is $20.2 \%$, which is reduced to $18.0 \%$ by increasing $T$ to $740 \mathrm{~ms}$. Further increasing $T$ does not reduce the error. State-ofthe-art algorithms provide refined feature vectors to improve classification. Combining MFCCs with stabilized modulation spectra and performing linear discriminant analysis, [8] obtains an error of $9.4 \%$, the best non-scattering result so far. A deep belief network trained on spectrograms [18], achieves $15.7 \%$ error with an SVM classifier. A sparse representation on a constant-Q transform [30], gives $16.6 \%$ error with an SVM.

Table III gives classification errors for different scattering feature vectors. For $l=1$, they are composed of firstorder time scattering coefficients computed with $Q_{1}=8$ and $T=740 \mathrm{~ms}$. These vectors are similar to MFCCs as shown by 11 . As a result, the classification error of $19.1 \%$ is close to that of MFCCs for the same $T$. For $l=2$, we add secondorder coefficients computed with $Q_{2}=2$. It reduces the error to $10.7 \%$. This $40 \%$ error reduction shows the importance of second-order coefficients for relatively large $T$. Third-order coefficients are also computed with $Q_{3}=1$. For $l=3$, including these coefficients reduces the error marginally to $10.6 \%$, at a significant computational and memory cost. We therefore restrict ourselves to $l=2$.

Musical genre recognition is a task which is partly invariant to frequency transposition. Incorporating a scattering along the $\log$-frequency variable, for frequency transposition invariance, reduces the error by about $15 \%$. These errors are obtained with a first-order scattering along log-frequency. Adding secondorder coefficients only improves results marginally.

Providing adaptivity for the wavelet octave bandwidth $Q_{1}$ by computing scattering coefficients for both $Q_{1}=1$ and $Q_{1}=8$ further reduces the error by almost $10 \%$. Indeed, music signals include both sharp transients and narrow-bandwidth frequency components. We thus have an error rate of $8.6 \%$, which compares favorably to the non-scattering state-of-the-art of $9.4 \%$ error [8].

Replacing the SVM with more sophisticated classifiers can improve results. A sparse representation classifier applied to second-order time scattering coefficients reduces the error rate from $10.7 \%$ to $8.8 \%$, as shown in [44]. Let us mention that the GTZAN database suffers from some significant statistical issues [45], which probably does not make it appropriate to evaluate further algorithmic refinements.

\section{Phone Segment Classification}

The same scattering representation is tested for phone segment classification with the TIMIT corpus [33]. The dataset contains 6300 phrases, each annotated with the identities, locations, and durations of its constituent phones. This task is simpler than continuous speech recognition, but provides an evaluation of scattering feature vectors for representing phone segments. Given the location and duration of a phone segment, the goal is to determine its class according to the standard protocol [46], [47]. The 61 phone classes (excluding the glottal stop /q/) are collapsed into 48 classes, which are used to train and test models. To calculate the error rate, these classes are then mapped into 39 clusters. Training is achieved on the full 3696-phrase training set, excluding "SA" sentences. The Gaussian kernel SVM parameters are optimized by validation on the standard 400-phrase development set [48]. The error is then calculated on the core 192-phrase test set.

An audio segment of length $192 \mathrm{~ms}$ centered on a phone can be represented as an array of MFCC feature vectors with half-overlapping time windows of duration $T$. This array, with the logarithm of the phone duration added, is fed to the SVM. In many cases, hidden Markov models or fixed time dilations are applied to match different MFCC sequences, to account for the time-warping of the phone segment [46], [47]. Table II shows that $T=23 \mathrm{~ms}$ yields a $18.5 \%$ error which is much less than the $60.5 \%$ error for $T=740 \mathrm{~ms}$. Indeed, many phones have a short duration with highly transient structures and are not well-represented by wide time windows.

A lower error of $17.1 \%$ is obtained by replacing the SVM with a sparse representation classifier on MFCC-like spectral features [49]. Combining MFCCs of different window sizes and using a committee-based hierarchical discriminative classifier, [43] achieves an error of $16.7 \%$, the best so far. Finally, convolutional deep-belief networks cascades convolutions, similarly to scattering, on a spectrogram using filters learned from the training data. These, combined with MFCCs, yield an error of $19.7 \%$ [13].

Rows 4 through 6 of Table II gives the classification results obtained by replacing MFCC vectors with a time scattering transform computed using first-order wavelets with $Q_{1}=8$. In order to retain local amplitude structure while creating invariance to loudness changes, first-order coefficients are renormalized in 33 using $|x|$ averaged over a window the size of the whole phone segment. Second- and third-order scattering coefficients are calculated with $Q_{2}=Q_{3}=1$. The 
best results are obtained with $T=32 \mathrm{~ms}$. For $l=1$, we only keep first-order scattering coefficients and get a $19.0 \%$ error, similar to that of MFCCs. The error is reduced by about $10 \%$ with $l=2$, a smaller improvement than for GTZAN because scattering invariants are computed on smaller time interval $T=32 \mathrm{~ms}$ as opposed to $740 \mathrm{~ms}$ for music. Second-order coefficients carry less energy when $T$ is smaller, as shown in Table II. For the same reason, third-order coefficients provide even less information compared to the GTZAN case, and do not improve results.

Note that no explicit time warping is needed in this model. Thanks to the scattering deformation stability, supervised linear classifiers can indeed compute time-warping invariants which remain sufficiently informative.

For $l=2$, cascading a log-frequency transposition invariance computed with a first-order frequency scattering transform of Section VII reduces the error by about $5 \%$. Computing a second-order frequency scattering transform only marginally improves results. Allowing to adapt the wavelet frequency resolution by computing scattering coefficients with $Q_{1}=1$ and $Q_{1}=8$ also reduces the error by about $5 \%$

Again, these results are for the problem of phone classification, where boundaries are given. Future work will concentrate on the task of phone recognition, where such information is absent. Since this task is more complex, performance is generally obtained worse, with the state-of-the-art achieved with a $17.7 \%$ error rate [16].

\section{CONCLUSION}

The success of MFCCs for audio classification can partially be explained by their stability to time-warping deformation. Scattering representations extend MFCCs by recovering lost high frequencies through successive wavelet convolutions. Over windows of $T \approx 200 \mathrm{~ms}$, signals recovered from first- and second-order scattering coefficients have a good audio quality. Normalized scattering coefficients characterizes amplitude modulations, and are stable to time-warping deformations. A frequency transposition invariant representation is obtained by cascading a second scattering transform along frequencies. Time and frequency scattering feature vectors yield state-of-the-art classification results with a Gaussian kernel SVM, for musical genre classification on GTZAN, and phone segment classification on TIMIT.

\section{APPENDIX}

Following (41), $a(t)$ is nearly constant over the time support of $\psi_{\lambda_{1}}$ and $\widehat{h}(\omega)$ is nearly constant over the frequency support of $\widehat{\psi}_{\lambda_{1}}$. It results that

$$
\left|x \star \psi_{\lambda_{1}}(t)\right| \approx\left|\widehat{h}\left(\lambda_{1}\right)\right|\left|e \star \psi_{\lambda_{1}}(t)\right| a(t) .
$$

Let $e(t)$ be a harmonic excitation. Since we supposed that $\lambda_{1} / Q_{1} \leq \xi, \widehat{\psi}_{\lambda_{1}}$ covers at most one harmonic whose frequency $k \xi$ is close to $\lambda_{1}$. It then results from (49) that

$$
\left|x \star \psi_{\lambda_{1}}(t)\right| \approx\left|\widehat{h}\left(\lambda_{1}\right)\right|\left|\widehat{\psi}_{\lambda_{1}}(k \xi)\right| a(t) .
$$

Computing $S_{1} x\left(t, \lambda_{1}\right)=\left|x \star \psi_{\lambda_{1}}\right| \star \phi(t)$ gives

$$
S_{1} x\left(t, \lambda_{1}\right) \approx\left|\widehat{h}\left(\lambda_{1}\right)\right|\left|\widehat{\psi}_{\lambda_{1}}(k \xi)\right| a \star \phi(t) .
$$

Let us compute

$$
\begin{aligned}
|x| \star \phi(t)= & \int|e \star h(u)| a(u) \phi(t-u) d u \\
= & \frac{2 \pi}{\xi} \int \sum_{n=-\infty}^{+\infty}|h(u+2 n \pi / \xi)| a(u) \phi(t-u) d u \\
= & \frac{2 \pi}{\xi} \sum_{k=-\infty}^{\infty} \int_{0}^{2 \pi / \xi} \sum_{n=-\infty}^{+\infty}|h(u+2 n \pi / \xi)| \\
& a(u+2 k \pi / \xi) \phi(t-u-2 k \pi / \xi) d u .
\end{aligned}
$$

Since $a(t)$ and $\phi(t)$ are approximately constant over intervals of size $2 \pi / \xi$, and the support of $h$ is smaller than $2 \pi / \xi$, one can verify that

$$
|x| \star \phi(t) \approx\|h\|_{1} a \star \phi(t) .
$$

This approximation together with (51) verifies (42).

It also results from 50 that

$$
S_{2} x\left(t, \lambda_{1}, \lambda_{2}\right) \approx\left|\widehat{h}\left(\lambda_{1}\right)\right|\left|\widehat{\psi}_{\lambda_{1}}(k \xi)\right|\left|a \star \psi_{\lambda_{2}}\right| \star \phi(t),
$$

which, combined with (51), yields (43).

Let us now consider a Gaussian white noise excitation $e(t)$. We saw in (49) that

$$
\left|x \star \psi_{\lambda_{1}}(t)\right| \approx\left|\widehat{h}\left(\lambda_{1}\right)\right|\left|e \star \psi_{\lambda_{1}}(t)\right| a(t) .
$$

Let us decompose

$$
\left|e \star \psi_{\lambda_{1}}(t)\right|=\mathbb{E}\left(\left|e \star \psi_{\lambda_{1}}\right|\right)+\epsilon(t)
$$

where $\epsilon(t)$ is a zero-mean stationary process. If $e(t)$ is a normalized Gaussian white noise then $e \star \psi_{\lambda_{1}}(t)$ is a Gaussian random variable of variance $\left\|\psi_{\lambda_{1}}\right\|^{2}$. It results that $\left|e \star \psi_{\lambda_{1}}(t)\right|$ and $\epsilon(t)$ have a Rayleigh distribution, and since $\psi$ is a complex wavelet with quadrature phase, one can verify that

$$
\mathbb{E}\left(\left|e \star \psi_{\lambda_{1}}\right|\right)^{2}=\frac{\pi}{4} \mathbb{E}\left(\left|e \star \psi_{\lambda_{1}}\right|^{2}\right)=\frac{\pi}{4}\left\|\psi_{\lambda_{1}}\right\|^{2} .
$$

Inserting 53 and this equation in 52 shows that

$$
\left|x \star \psi_{\lambda_{1}}(t)\right| \approx\left|\widehat{h}\left(\lambda_{1}\right)\right|\left(\pi^{1 / 2} 2^{-1}\left\|\psi_{\lambda_{1}}\right\| a(t)+a(t) \epsilon(t)\right) .
$$

When averaging with $\phi$, we get

$S_{1} x\left(t, \lambda_{1}\right) \approx\left|\widehat{h}\left(\lambda_{1}\right)\right|\left(\pi^{1 / 2} 2^{-1}\left\|\psi_{\lambda_{1}}\right\| a \star \phi(t)+(a \epsilon) \star \phi(t)\right)$.

Suppose that $a(t)$ is not sparse, in the sense that

$$
\frac{|a|^{2} \star \phi(t)}{|a \star \phi|^{2}(t)} \sim 1
$$

It means that ratios between local $\mathbf{L}^{2}$ and $\mathbf{L}^{1}$ norms of $a$ is of the order of 1 . We are going to show that if $T^{-1} \ll \lambda_{1} Q_{1}^{-1}$ then

$$
\frac{\mathbb{E}\left(|(a \epsilon) \star \phi(t)|^{2}\right)}{\left\|\psi_{\lambda_{1}}\right\|^{2}|a \star \phi(t)|^{2}} \ll 1
$$

which implies

$$
S_{1} x\left(t, \lambda_{1}\right) \approx \frac{\pi^{1 / 2}}{2}\|\psi\| \lambda_{1}{ }^{1 / 2}\left|\widehat{h}\left(\lambda_{1}\right)\right| a \star \phi(t) .
$$

We give the main arguments to compute the order of magnitudes of the stochastic terms, but it is not a rigorous proof. 
For a detailed argument, see [50]. Computations rely on the following lemma.

Lemma 1. Let $z(t)$ be a zero-mean stationary process of power spectrum $\widehat{R}_{z}(\omega)$. For any deterministic functions $a(t)$ and $h(t)$

$$
\mathbb{E}\left(|(z a) \star h(t)|^{2}\right) \leq \sup _{\omega} \widehat{R}_{z}(\omega)|a|^{2} \star|h|^{2}(t) .
$$

Proof: Let $R_{z}(\tau)=\mathbb{E}(z(t) z(t+\tau))$,

$\mathbb{E}\left(|(z a) \star h(t)|^{2}\right)=\iint R_{z}(v-u) a(u) h(t-u) a(v)^{*} h(t-v)^{*} d u d w h$ here

and hence

$$
\mathbb{E}\left(|(z a) \star h(t)|^{2}\right)=\left\langle R_{z} y_{t}, y_{t}\right\rangle \text { with } y_{t}(u)=a(u) h(t-u) .
$$

Since $R_{z}$ is the kernel of a positive symmetric operator whose spectrum is bounded by $\sup _{\omega} \widehat{R}_{z}(\omega)$ it results that

$$
\mathbb{E}\left(|(z a) \star h(t)|^{2}\right) \leq \sup _{\omega} \widehat{R}_{z}(\omega)\left\|y_{t}\right\|^{2}=\sup _{\omega} \widehat{R}_{z}(\omega)|a|^{2} \star|h|^{2}(t) .
$$

Because $e(t)$ is a normalized white noise, with a Gaussian chaos expansion, one can verify [50] that $\sup _{\omega} \widehat{R}_{\epsilon}(\omega) \leq C(1-$ $\pi / 4$ ), where $C=\|\psi\|_{1}^{2} \approx 1$. Applying Lemma 1 to $z=\epsilon$ and $h=\phi$ gives

$$
\mathbb{E}\left(|(\epsilon a) \star \phi(t)|^{2}\right) \leq(1-\pi / 4)|a|^{2} \star|\phi|^{2}(t) .
$$

Since $\phi$ has a duration $T$, it can be written as $\phi(t)=$ $T^{-1} \phi_{0}\left(T^{-1} t\right)$ for some $\phi_{0}$ of duration 1 . As a result, if (56) holds then

$$
\frac{|a|^{2} \star|\phi|^{2}(t)}{|a \star \phi(t)|^{2}} \sim \frac{1}{T}
$$

The frequency support of $\psi_{\lambda_{1}}$ is proportional to $\lambda_{1} Q_{1}^{-1}$, so we have $\left\|\psi_{\lambda_{1}}\right\|^{2} \sim \lambda_{1} Q_{1}^{-1}$. Together with (60), if $T^{-1} \ll \lambda_{1} Q_{1}^{-1}$ it proves (57) which yields (58).

We approximate $|x| \star \phi(t)$ similarly. First, we write

$$
|e \star h(t)|=\mathbb{E}|e \star h|+\epsilon^{\prime}(t),
$$

where $\epsilon^{\prime}(t)$ is a zero-mean stationary process. Since $e \star h(t)$ is normally distributed in $\mathbb{R},|e \star h|(t)$ has $\chi^{1}$ distribution and

$$
\mathbb{E}(|e \star h|)^{2}=\frac{2}{\pi} \mathbb{E}\left(|e \star h|^{2}\right)=\frac{2}{\pi}\|h\|^{2},
$$

which then gives

$$
|x| \star \phi(t)=\sqrt{\frac{2}{\pi}}\|h\| a \star \phi(t)+\left(a \epsilon^{\prime}\right) \star \phi(t) .
$$

One can show that $\sup _{\omega} \widehat{R}_{\epsilon^{\prime}}(\omega) \leq(1-2 / \pi)\|h\|_{1}^{2}$ [50], so applying Lemma 1 gives

$$
\mathbb{E}\left(\left|\left(a \epsilon^{\prime}\right) \star \phi(t)\right|^{2}\right) \leq(1-2 / \pi)\|h\|_{1}^{2}|a|^{2} \star|\phi|^{2}(t) .
$$

Now (60) implies that

$$
\frac{\mathbb{E}\left(\left|\left(a \epsilon^{\prime}\right) \star \phi(t)\right|^{2}\right)}{\|h\|^{2}|a \star \phi(t)|^{2}} \ll 1
$$

since $a$ is non-sparse and because $h$ has a support much smaller than $T$ so $\|h\|_{1}^{2} /\|h\|^{2} \ll T$. Consequently,

$$
|x| \star \phi(t) \approx \sqrt{\frac{2}{\pi}}\|h\| a \star \phi(t),
$$

which, together with (58) gives (44).

Let us now compute $S_{2} x\left(t, \lambda_{1}, \lambda_{2}\right)=|| x \star \psi_{\lambda_{1}}\left|\star \psi_{\lambda_{2}}\right| \star \phi(t)$. If $T^{-1} \ll \lambda_{1} Q_{1}^{-1}$ then (58) together with (54) shows that

$$
\begin{gathered}
\frac{S_{2} x\left(t, \lambda_{1}, \lambda_{2}\right)}{S_{1} x\left(t, \lambda_{1}\right)} \approx \frac{\left|a \star \psi_{\lambda_{2}}\right| \star \phi(t)}{a \star \phi(t)}+\widetilde{\epsilon}(t), \\
0 \leq \widetilde{\epsilon}(t) \leq \frac{2\left|(a \epsilon) \star \psi_{\lambda_{2}}\right| \star \phi(t)}{\pi^{1 / 2}\left\|\psi_{\lambda_{1}}\right\| a \star \phi(t)} .
\end{gathered}
$$

Observe that

$$
E\left(\left|(a \epsilon) \star \psi_{\lambda_{2}}\right| \star \phi(t)\right) \leq E\left(\left|(a \epsilon) \star \psi_{\lambda_{2}}\right|^{2}\right)^{1 / 2} \star \phi(t) .
$$

Lemma 1 applied to $z=\epsilon$ and $h=\psi_{\lambda_{2}}$ gives the following upper bound:

$$
\mathbb{E}\left(\left|(a \epsilon) \star \psi_{\lambda_{2}}(t)\right|^{2}\right) \leq C(1-\pi / 4)|a|^{2} \star\left|\psi_{\lambda_{2}}\right|^{2}(t) .
$$

One can write $\left|\psi_{\lambda_{2}}(t)\right|=\lambda_{2} Q_{2}^{-1} \theta\left(\lambda_{2} Q_{2}^{-1} t\right)$ where $\theta(t)$ satisfies $\int \theta(t) d t \sim 1$. Similarly to (60), if (56) holds over time intervals of size $Q_{2} / \lambda_{2}$, then

$$
\frac{|a|^{2} \star\left|\psi_{\lambda_{2}}\right|^{2}(t)}{\left.|a \star| \psi_{\lambda_{2}}\right|^{2}} \sim \frac{\lambda_{2}}{Q_{2}} .
$$

Since $\left\|\psi_{\lambda_{1}}\right\|^{2} \sim \lambda_{1} Q_{1}^{-1}$ and $\left|\psi_{\lambda_{2}}\right| \star \phi(t) \sim \phi(t)$ when $Q_{2} / \lambda_{2} \leq T$, it results from 686970 that $0 \leq \mathbb{E}(\widetilde{\epsilon}(t)) \leq$ $C(4 / \pi-1)^{1 / 2}\left(\lambda_{2} Q_{1}\right)^{1 / 2}\left(\lambda_{1} Q_{2}\right)^{-1 / 2}$ with $C \sim 1$.

\section{REFERENCES}

[1] V. Chudáček, J. Andén, S. Mallat, P. Abry, and M. Doret, "Scattering transform for intrapartum fetal heart rate characterization and acidosis detection," in Proc. IEEE EMBC, 2013.

[2] H. Hermansky, "The modulation spectrum in the automatic recognition of speech," in Proc. IEEE ASRU, 1997, pp. 140-147.

[3] M. S. Vinton and L. E. Atlas, "Scalable and progressive audio codec," in Acoustics, Speech, and Signal Processing, 2001. Proceedings.(ICASSP'01). 2001 IEEE International Conference on, vol. 5. IEEE, 2001, pp. 3277-3280.

[4] J. McDermott and E. Simoncelli, "Sound texture perception via statistics of the auditory periphery: Evidence from sound synthesis," Neuron, vol. 71, no. 5, pp. 926-940, 2011.

[5] M. Ramona and G. Peeters, "Audio identification based on spectral modeling of bark-bands energy and synchronization through onset detection," in Proc. IEEE ICASSP, 2011, pp. 477-480.

[6] M. Slaney and R. Lyon, Visual representations of speech signals. M. Cooke, S. Beet and M. Crawford (Eds.) John Wiley and Sons, 1993, ch. On the importance of time-a temporal representation of sound, pp. 95-116.

[7] R. D. Patterson, "Auditory images: How complex sounds are represented in the auditory system," Journal of the Acoustical Society of Japan (E), vol. 21, no. 4, pp. 183-190, 2000.

[8] C. Lee, J. Shih, K. Yu, and H. Lin, "Automatic music genre classification based on modulation spectral analysis of spectral and cepstral features," IEEE Transactions on Multimedia, vol. 11, no. 4, pp. 670-682, 2009.

[9] D. Ellis, X. Zeng, and J. McDermott, "Classifying soundtracks with audio texture features," in Proc. IEEE ICASSP, Prague, Czech Republic, May. 22-27 2011, pp. 5880-5883.

[10] J. K. Thompson and L. E. Atlas, "A non-uniform modulation transform for audio coding with increased time resolution," in Acoustics, Speech, and Signal Processing, 2003. Proceedings.(ICASSP'03). 2003 IEEE International Conference on, vol. 5. IEEE, 2003, pp. V-397.

[11] S. Mallat, "Group invariant scattering," Commun. Pure Appl. Math., vol. 65, no. 10, pp. 1331-1398, 2012. 
[12] Y. LeCun, K. Kavukvuoglu, and C. Farabet, "Convolutional networks and applications in vision," in Proc. IEEE ISCAS, 2010.

[13] H. Lee, P. Pham, Y. Largman, , and A. Ng, "Unsupervised feature learning for audio classification using convolutional deep belief networks," in Proc. NIPS, 2009.

[14] G. Hinton, L. Deng, D. Yu, G. E. Dahl, A.-R. Mohamed, N. Jaitly, A. Senior, V. Vanhoucke, P. Nguyen, T. N. Sainath et al., "Deep neural networks for acoustic modeling in speech recognition: The shared views of four research groups," Signal Processing Magazine, IEEE, vol. 29, no. 6, pp. 82-97, 2012.

[15] L. Deng, O. Abdel-Hamid, and D. Yu, "A deep convolutional neural network using heterogeneous pooling for trading acoustic invariance with phonetic confusion," in Proc. ICASSP, 2013.

[16] A. Graves, A.-R. Mohamed, and G. Hinton, "Speech recognition with deep recurrent neural networks," Proc. ICASSP, 2013.

[17] E. J. Humphrey, T. Cho, and J. P. Bello, "Learning a robust tonnetz-space transform for automatic chord recognition," in Proc. IEEE ICASSP, 2012, pp. 453-456.

[18] P. Hamel and D. Eck, "Learning features from music audio with deep belief networks," in Proc. ISMIR, 2010.

[19] E. Battenberg and D. Wessel, "Analyzing drum patterns using conditional deep belief networks," in Proc. ISMIR, 2012.

[20] T. Dau, B. Kollmeier, and A. Kohlrausch, "Modeling auditory processing of amplitude modulation. I. Detection and masking with narrow-band carriers," J. Acoust. Soc. Am., vol. 102, no. 5, pp. 2892-2905, 1997.

[21] T. Chi, P. Ru, and S. Shamma, "Multiresolution spectrotemporal analysis of complex sounds," J. Acoust. Soc. Am., vol. 118, no. 2, pp. 887-906, 2005.

[22] N. Mesgarani, M. Slaney, and S. A. Shamma, "Discrimination of speech from nonspeech based on multiscale spectro-temporal modulations," IEEE Audio, Speech, Language Process., vol. 14, no. 3, pp. 920-930, 2006.

[23] J. Bruna and S. Mallat, "Invariant scattering convolution networks," IEEE Trans. Pattern Anal. Mach. Intell., vol. 35, no. 8, pp. 1872-1886, 2013.

[24] L. Sifre and S. Mallat, "Rotation, scaling and deformation invariant scattering for texture discrimination," in Proc. CVPR, 2013.

[25] S. Mallat, A wavelet tour of signal processing. Academic Press, 1999.

[26] S. Schimmel and L. Atlas, "Coherent envelope detection for modulation filtering of speech," in Proc. of ICASSP, vol. 1, 2005, pp. 221-224.

[27] R. Turner and M. Sahani, "Probabilistic amplitude and frequency demodulation," in Advances in Neural Information Processing Systems, 2011, pp. 981-989.

[28] G. Sell and M. Slaney, "Solving demodulation as an optimization problem," Audio, Speech, and Language Processing, IEEE Transactions on, vol. 18, no. 8, pp. 2051-2066, 2010.

[29] I. Waldspurger and S. Mallat, "Recovering the phase of a complex wavelet transform," CMAP, Ecole Polytechnique, Tech. Rep., 2012

[30] M. Henaff, K. Jarrett, K. Kavukcuoglu, and Y. LeCun, "Unsupervised learning of sparse features for scalable audio classification," in Proc. ISMIR, 2011.

[31] J. Nam, J. Herrera, M. Slaney, and J. Smith, "Learning sparse feature representations for music annotation and retrieval," in Proc. ISMIR, 2012.

[32] E. C. Smith and M. S. Lewicki, "Efficient auditory coding," Nature, vol. 439, no. 7079, pp. 978-982, 2006.

[33] W. Fisher, G. Doddington, and K. Goudie-Marshall, "The DARPA speech recognition research database: specifications and status," in Proc. DARPA Workshop on Speech Recognition, 1986, pp. 93-99.

[34] L. Lucy, "An iterative technique for the rectification of observed distributions," Astron. J., vol. 79, p. 745, 1974.

[35] E. J. Candès, Y. C. Eldar, T. Strohmer, and V. Voroninski, "Phase retrieval via matrix completion," SIAM Journal on Imaging Sciences, vol. 6, no. 1, pp. 199-225, 2013.

[36] I. Waldspurger, A. d'Aspremont, and S. Mallat, "Phase recovery, maxcut and complex semidefinite programming," CMAP, Ecole Polytechnique, Tech. Rep., 2012.

[37] D. W. Griffin and J. S. Lim, "Signal estimation from modified shorttime fourier transform," IEEE Trans. Acoust., Speech, Signal Process., vol. 32, no. 2, pp. 236-243, 1984.

[38] S. Davis and P. Mermelstein, "Comparison of parametric representations for monosyllabic word recognition in continuously spoken sentences," IEEE Trans. Acoust., Speech, Signal Process., vol. 28, no. 4, pp. 357366,1980

[39] C.-C. Chang and C.-J. Lin, "LIBSVM: A library for support vector machines," ACM Transactions on Intelligent Systems and Technology, vol. 2, pp. 27:1-27:27, 2011, software available at http://www.csie.ntu. edu.tw/ cjlin/libsvm

[40] G. Tzanetakis and P. Cook, "Musical genre classification of audio signals," IEEE Transactions on Speech and Audio Processing, vol. 10, no. 5, pp. 293-302, 2002.

[41] J. Andén and S. Mallat, "Multiscale scattering for audio classification," in Proc. ISMIR, Miami, Florida, Unites States, Oct. 24-28 2011, pp. 657-662.

[42] C. Baugé, M. Lagrange, J. Andén, and S. Mallat, "Representing environmental sounds using the separable scattering transform," in Proc. IEEE ICASSP, 2013.

[43] H.-A. Chang and J. R. Glass, "Hierarchical large-margin gaussian mixture models for phonetic classification," in Proc. IEEE ASRU. IEEE, 2007, pp. 272-277.

[44] X. Chen and P. J. Ramadge, "Music genre classification using multiscale scattering and sparse representations," in Proc. CISS, 2013.

[45] B. L. Sturm, "An analysis of the GTZAN music genre dataset," in Proceedings of the second international ACM workshop on Music information retrieval with user-centered and multimodal strategies. ACM, 2012, pp. 7-12.

[46] K.-F. Lee and H.-W. Hon, "Speaker-independent phone recognition using hidden markov models," Acoustics, Speech and Signal Processing, IEEE Transactions on, vol. 37, no. 11, pp. 1641-1648, 1989.

[47] P. Clarkson and P. J. Moreno, "On the use of support vector machines for phonetic classification," in IEEE Trans. Acoust., Speech, Signal Process., vol. 2. IEEE, 1999, pp. 585-588.

[48] A. K. Halberstadt, "Heterogeneous acoustic measurements and multiple classifiers for speech recognition," Ph.D. dissertation, Massachusetts Institute of Technology, 1998.

[49] T. N. Sainath, D. Nahamoo, D. Kanevsky, B. Ramabhadran, and P. Shah, "A convex hull approach to sparse representations for exemplar-based speech recognition," in Proc. IEEE ASRU. IEEE, 2011, pp. 59-64.

[50] J. Andén, "Time and frequency scattering for audio classification," Ph.D. dissertation, Ecole Polytechnique, 2014. 